Received: 29.11 .2018

Revised: 25.01 .2019

Accepted: 15.02 .2019

DOI: $10.17804 / 2410-9908.2019 .1 .006-042$

\title{
TEMPERATURE FIELD INVESTIGATION IN LAYERED FLOWS OF A VERTICALLY SWIRLING VISCOUS INCOMPRESSIBLE FLUID UNDER TWO THERMOCAPILLAR FORCES AT A FREE BOUNDARY
}

\author{
N. V. Burmasheva ${ }^{\text {a)* }}$ and E. Yu. Prosviryakov ${ }^{\text {b) }}$ \\ Institute of Engineering Science, Ural Branch of the Russian Academy of Sciences, \\ 34 Komsomolskaya St., Ekaterinburg, 620049, Russian Federation \\ a) (iD https://orcid.org/0000-0003-4711-1894 nat_burm@mail.ru; \\ b) iD https://orcid.org/0000-0002-2349-7801 ख evgen_pros@mail.ru \\ *Corresponding author. E-mail: nat_burm@mail.ru \\ Address for correspondence: 34 Komsomolskaya St., Ekaterinburg, 620049, Russian Federation \\ Tel.: +7 (343) 37535 76; fax: +7 (343) 3745330
}

The exact solution of the Oberbeck-Boussinesq equations, which describes the influence of the thermocapillary effect on the convective flows of a viscous incompressible fluid, is discussed. The class of exact solutions is chosen in such a way that it allows one to solve an overdetermined system of equations for the motion of a fluid by identically satisfying the incompressibility condition. Heat sources at both boundaries, which heat (cool) the fluid, are selected as the boundary conditions. The exact solution has been obtained and the temperature field has been studied. It is shown that the structure of the exact solution does not allow one to reduce the dimension of the boundary value problem under study. It is also shown that the obtained exact solution admits the presence of countercurrents in the fluid.

Keywords: layered flow, exact solution, counterflows, Marangoni convection, vertical swirl, vortex flow, system of Oberbeck-Boussinesq equations.

\section{References}

1. Gershuni G.Z., Zhukhovitskii E.M. Convective Stability of Incompressible Fluids. Israel Program for Scientific Translations. Jerusalem, Keter Publishing House, 1976, 330 p.

2. Ekman V.W. On the Influence of the Earth's Rotation on Ocean-Currents. Ark. Mat. Astron. Fys., 1905, vol. 2, no. 11, pp. 1-52.

3. Hiemenz K. Die Grenzschicht an einem in den gleichfurmigen Flıssigkeitsstrom eingetauchten geraden Kreiszylinder. Dingler's Politech. J., 1911, vol. 326, pp. 321-324.

4. Ekman V.W. Uber Horizontazirkulation bei winder-reugten Meeresstromungen. Arkiv Mat., Astr., Phys., 1923, vol. 17, no. 26, pp. 1-74.

5. Pedlosky J. Geophysical Fluid Dynamics. New York, Heidelberg, Berlin, Springer-Verlag, 1987, $710 \mathrm{p}$.

6. Haeusser T.M., Leibovich S. Pattern formation in the marginally unstable Ekman layer. J. Fluid Mech., 2003, vol. 479, pp. 125-144. DOI: 10.1017/S0022112002003415.

7. Schwarz K.G. Stability of thermocapillary advective flow in a slowly rotating liquid layer under microgravity conditions. Fluid Dynamics, 2012, vol. 47, iss. 1, pp. 37-49. DOI: $10.1134 /$ S001546281201005X.

8. Aristov S.N., Frik P.G. Nonlinear effects of the Ekman layer on the dynamics of largescale eddies in shallow water. J. Appl. Mech. Tech. Phys., 1991, vol. 32, no. 2, pp. 189-194. 
9. Gorshkov A.V., Prosviryakov E.Y. Ekman Convective Layer Flow of a Viscous Incompressible Fluid. Izvestiya. Atmospheric and Oceanic Physics, 2018, vol. 54, no. 2, pp. 189-195. DOI: $10.1134 / \mathrm{S} 0001433818020081$.

10. Kryukov N.D., Shmatkov V.A. A review of studies of wind-generated surface currents. Eurasian Union of Scientists, 2015, № 6-2 (15), pp. 109-113. (In Russian).

11. Shvarts K.G. On an exact solution of the Navier-Stokes equations describing non-isothermal large-scale flow in a rotating fluid layer with a free upper boundary. Vestnik Permskogo Universiteta. Matematika. Mekhanika. Informatika, 2016, no. 2 (33), pp. 118-124. (In Russian).

12. Shvarts K.G. Exact solution of the Navier-Stokes equation describing nonisothermal largescale flows in a rotating layer of liquid with free upper surface. J. Math. Sci., 2018, vol. 230, no. 5, pp. 813-817. DOI: 10.1007/s10958-018-3796-y.

13. Brown R. A. Analytical Methods in Planetary Boundary-Layer Modelling. Hilger, 1974.

14. Welander P. The thermocline problem. Phil. Trans. R. Soc. Lond. A., 1971, vol. 270, pp. 415-421. DOI: 10.1098/rsta.1971.0081.

15. Chefranov S.G. Cyclone-anticyclone vortex asymmetry mechanism and linear Ekman friction. Journal of Experimental and Theoretical Physics, 2016, vol. 122, no. 4, pp. 759-768. DOI: $10.1134 / \mathrm{S} 1063776116040038$.

16. Riabouchinsky D. Quelques considerations sur les mouvements plans rotationnels d'un liquid. C. R. Hebdomadaires Acad. Sci., 1924, vol. 179, pp. 1133-1136.

17. Galaktionov V.A., Svirshchevskii S.R. Exact solutions and invariant subspaces of nonlinear partial differential equations in mechanics and physics. Boca Raton, Chapman and Hall/CRC, 2007. DOI: 10.1201/9781420011623.

18. Pukhnachev V.V. Group Properties of the Navier-Stokes Equations in a Plane Case. Prikl. Mekh. Tekh. Fiz., no. 1, pp. 83-90. (In Russian).

19. Atlas osesimmetrichnykh kavitatsionnykh techeniy tipa Ryabushinskogo [Atlas of Axisymmetric Cavitation Ryabushinsky-Type Flows]. Novosibirsk, Institut Gidrodinamiki SO AN SSSR, 1968. (In Russian).

20. Guzevsky L.G. Osesimmetrichnye zadachi obtekaniya so svobodnymi granitsami. In: Issledovaniya po razvitoy kavitatsii [Advanced Cavitation Research]. Novosibirsk, Izdatelstvo SO AN SSSR, 1976. (In Russian).

21. Aristov S. N., Knyazev D.V. Viscous fluid flow between moving parallel plates. Fluid Dynamics, 2012, vol. 47, iss. 4, pp 476-482. DOI: 10.1134/S0015462812040060.

22. Petrov A.G. Exact solution of the Navier-Stokes equations in a fluid layer between the moving parallel plates. Journal of Applied Mechanics and Technical Physics, 2012, vol. 53, no. 5, pp. 642-646. DOI: 10.1134/S0021894412050021.

23. Vlasova S.S., Prosviryakov E.Yu. Two-dimensional convection of an incompressible viscous fluid with the heat exchange on the free border. Vestn. Samar. Gos. Tekh. Univ., Ser. Fiz.-Mat. Nauki, 2016, vol. 20, no. 3, pp. 567-577. DOI: https://doi.org/10.14498/vsgtu1483. (In Russian).

24. Aristov S.N., Prosviryakov E.Yu. On one class of analytic solutions of the stationary axisymmetric convection Benard-Maragoni viscous incompreeible fluid. Vestn. Samar. Gos. Tekh. Univ., Ser. Fiz.-Mat. Nauki, 2013, vol. 3, no. 32, pp. 110-118. DOI: 10.14498/vsgtu1205. (In Russian).

25. Aristov S.N., Privalova V., Prosviryakov E.Y. Stationary nonisothermal Couette flow. Quadratic heating of the upper boundary of the fluid layer. Rus. J. Nonlin. Dyn., 2016, vol. 12, no. 2, pp. 167-178. DOI: $10.20537 /$ nd1602001.

26. Fomin A.A, Fomina L.N. On the solution of fluid flow and heat transfer problem in a 2D channel with backward-facing step. Vestn. Samar. Gos. Tekh. Univ., Ser. Fiz.-Mat. Nauki, 2017, vol. 21, no. 2, pp. 362-375. DOI: 10.14498/vsgtu1545.

27. Shtern V. Counterflows. Paradoxical Fluid Mechanics Phenomena. Cambridge, Cambridge University Press, 2012. DOI: 10.1017/CBO9781139226516. 
28. Stuart J.T. The viscous flow near a stagnation point when the external flow has uniform vorticity. Journal of the Aerospace Sciences, 1959, vol. 26, no. 2, pp. 124-125. DOI: 10.2514/8.7963.

29. Dorrepaal J.M. An exact solution of the Navier-Stokes equation which describes nonorthogonal stagnation-point flow in two dimensions. J. Fluid Mech., 1986, vol. 163, no. 1, pp. 141-147. DOI: $10.1017 / \mathrm{s} 0022112086002240$.

30. Riesco-Chueca P., de la Mora J.F. Brownian motion far from equilibrium: a hypersonic approach. J. of Fluid Mech., 1990, vol. 214, pp. 639-663. DOI: 10.1017/ S0022112090000301.

31. Aristov S.N., Prosviryakov E.Yu. Nonuniform convective Couette flow. Fluid Dynamics, 2016, vol. 51, iss. 5, pp. 581-587. DOI: 10.1134/S001546281605001X.

32. Gorshkov A.V., Prosviryakov E.Yu. Layered Benard-Marangoni convection during heat transfer according to the Newton's law of cooling. Computer Research and Modeling, 2016, vol. 8, no. 6, pp. 927-940. (In Russian).

33. Aristov S.N., Prosviryakov E.Y., Spevak L.F. Nonstationary laminar thermal and solutal Marangoni convection of a viscous fluid. Computational Continuum Mechanics, 2015, vol. 8, no. 4, pp. 445-456. DOI: 10.7242/1999-6691/2015.8.4.38.

34. Burmasheva N.V., Prosviryakov E.Yu. A large-scale layered stationary convection of an incompressible viscous fluid under the action of shear stresses at the upper boundary. Velocity field investigation. Journal of Samara State Technical University, Ser. Physical and Mathematical Sciences, 2017, vol. 21, no. 1, pp. 180-196. DOI: 10.14498/vsgtu1527.

35. Burmasheva N.V., Prosviryakov E.Yu. Exact solutions for layered large-scale convection induced by tangential stresses specified on the free boundary of a fluid layer. IOP Conference Series: Materials Science and Engineering, 2017, vol. 208, conf. 1. DOI: 10.1088/1757899X/208/1/012010.

36. Burmasheva N.V., Prosviryakov E.Yu. Exact solutions for natural convection of layered flows of a viscous incompressible fluid with specified tangential forces and the linear distribution of temperature on the layer boundaries. Diagnostics, Resource and Mechanics of materials and structures, 2017, iss. 4, pp. 16-31. DOI: 10.17804/2410-9908.2017.4.016-031. Available at: http://dream-journal.org/DREAM_Issue_4_2017_Burmasheva_N.V._et_al._016_031.pdf

37. Burmasheva N.V., Prosviryakov E.Yu. Exact solution for the layered convection of a viscous incompressible fluid at specified temperature gradients and tangential forces on the free boundary. AIP Conference Proceedings, 2017, vol. 1915, iss. 1. DOI: 10.1063/1.5017353.

38. Burmasheva N.V., Prosviryakov E.Yu. A large-scale layered stationary convection of a incompressible viscous fluid under the action of shear stresses at the upper boundary. Temperature and presure field investigation. J. Samara State Tech. Univ., Ser. Phys. Math. Sci., 2017, vol. 21, no. 4, pp. 736-751. DOI: 10.14498/vsgtu1568. (In Russian).

39. Aristov S.N., Knyazev D.V., Polyanin A.D. Exact solutions of the Navier-Stokes equations with the linear dependence of velocity components on two space variables. Theoretical Foundations of Chemical Engineering, 2009, vol. 43, no. 5, pp. 642-662. DOI: 10.1134/S0040579509050066.

40. Ostroumov G.A. Free convection under the condition of the internal problem. Washington, NACA Technical Memorandum 1407, National Advisory Committee for Aeronautics, 1958.

41. Birikh R.V. Thermocapillary convection in a horizontal layer of liquid. J. Appl. Mech. Tech. Phys., 1966, vol. 7, no. 3, p. 43.

42. Napolitano L.G. Plane Marangoni-Poiseulle flow of two immiscible fluids. Acta Astronautica, 1980, vol. 7, no. 4, pp. 461-478.

43. Pukhnachev V.V. Non-stationary analogues of the Birikh solution. Izvestiya AltGU, 2011, nos. 1-2, pp. 62-69. (In Russian).

44. Goncharova O.N., Rezanova E.V. Example of an exact solution of the stationary problem of two-layer flows with evaporation at the interface. Journal of Applied Mechanics and Technical Physics, 2014, vol. 55, no. 2, pp. 247-257. DOI: 10.1134/s0021894414020072. 
45. Aristov S.N., Prosviryakov E.Y. On laminar flows of planar free convection. Rus. J. Nonlin. Dyn., 2013, vol. 9, no. 4, pp. 651-657. DOI: 10.20537/nd1304004.

46. Schwarz K.G. Plane-parallel advective flow in a horizontal incompressible fluid layer with rigid boundaries. Fluid Dynamics, 2014, vol. 49, iss. 4, pp. 438-442. DOI: 10.1134/S0015462814040036.

47. Bratsun D.A., Vyatkin V.A., Mukhamatullin A.R. On exact nonstationary solutions of equations of vibrational convection. Computational Continuum Mechanics, 2017, vol. 10, no. 4, pp. 433-444. DOI: 10.7242/1999-6691/2017.10.4.35.

48. Schwarz K.G. Plane parallel advective flow in a horizontal layer of incompressible fluid with an internal linear heat source. Prikladnaya Matematika i Mekhanika, 2018, vol. 82, no. 1, pp. 25-30. (In Russian).

49. Aristov S.N., Shvarts K.G. Convective heat transfer in a locally heated plane incompressible fluid layer. Fluid Dynamics, 2013, vol. 48, iss. 3, pp 330-335. DOI: 10.1134/S001546281303006X. 50. Gorshkov A.V., Prosviryakov E.Yu. Analytic solutions of stationary complex convection describing a shear stress field of different signs. In: Trudy instituta matematiki i mekhaniki UrO RAN [Proccedings of Institute of Mechanics and Mathematics of RAS, Ural Branch, 2017, vol. 23, no. 2, pp. 32-41]. (In Russian).

51. Rafiq Sh., Nawaz M., Mustahsan M. Casson Fluid Flow due to Non-Coaxial Rotation of a Porous Disk and the Fluid at Infinity Through a Porous Medium. Journal of Applied Mechanics and Technical Physics, 2018, vol. 59, iss. 4, pp. 601-607. DOI: 10.1134/S0021894418040053.

52. Andreev V.K. Resheniya Birikha uravneniy konvektsii $i$ nekotorye ego obobshcheniya: preprint [Birikh Solutions of Convection Equations and Some of its Generalizations: Preprint]. Krasnoyarsk, 2010, № 1-10. (In Russian).

53. Birikh R.V. Thermocapillary convection in a horizontal layer of liquid. J. Appl. Mech. Tech. Phys., 1966, vol. 7, no. 3, pp. 43-44.

54. Pukhnachev V.V. Group-theoretical methods in the convection problems. In: Application of Mathematics in Technical and Natural Sciences, M.D. Todorov and C.I. Christov, eds., American Institute of Physics, CP 1404, Melwille, NY, 2011, pp. 31-42.

55. Sidorov A.F. Two classes of solutions of the fluid and gas mechanics equations and their connection to traveling wave theory. Journal of Applied Mechanics and Technical Physics, 1989, vol. 30, iss. 2, pp. 197-203. DOI: 10.1007/BF00852164.

56. Aristov S.N., Shvarts K.G. Advective flow in a rotating liquid film. J. Appl. Mech. Tech. Phys., 2016, vol. 57, no. 1, pp. 188-194. DOI: 10.1134/S0021894416010211.

57. Aristov S.N., Prosviryakov E.Yu. A new class of exact solutions for three-dimensional thermal diffusion equations. Theor. Found. Chem. Eng., 2016, vol. 50, no. 3, pp. 286-293. DOI: 10.1134/S0040579516030027.

58. Aristov S.N., Frik P.G. Nonlinear effects of the Ekman layer on the dynamics of largescale eddies in shallow water. J. Appl. Mech. Tech. Phys., 1991, vol. 32, no. 2, pp. 189-194. DOI: $10.1007 / \mathrm{BF} 00858033$.

59. Aristov S.N., Shvarts K.G. Vikhrevye Techeniya Advektivnoy Prirody vo Vrashchayushchemsya Sloe Zhidkosti [Vortex Flows of Advective Nature in the Rotary Layer of Fluid]. Perm, Perm Univ. Publ., 2006. (In Russian).

60. Aristov S.N., Shvarts K.G. Vikhrevye Techeniya v Tonkikh Sloyakh Zhidkosti [Vortical Flows in Thin Fluid Layers]. Kirov, VyatGU, 2011, 207 pp. (In Russian).

61. Ingel L.Kh., Aristov S.N. The class of exact solutions of nonlinear problems on thermal circulation associated with volumetric heat release in the atmosphere. Tr. In-ta Eksperim. Meteorol., 1996, no. 27 (162), pp. 142-157. (In Russian).

62. Aristov S.N., Prosviryakov E.Yu. On laminar flows of planar free convection. Russian Journal of Nonlinear Dynamics, 2013, vol. 9, no. 4, pp. 651-657. DOI: 10.20537/nd1304004.

63. Andreev V.K., Gaponenko Ya.A., Goncharova O.N., Pukhnachev V.V. Mathematical Models of Convection. Berlin, Boston, De Gryuter Publ., 2012, 417 p. 
64. Bekezhanova V.B., Goncharova O.N. Evaporative convection problems (review). Prikladnaya Matematika i Mekhanika, 2018, vol. 82, no. 2, pp. 219-260. (In Russian).

65. Galkin V.A., Dubovik A.O. About Layered Flow Modeling of Magnetic Viscous Incompressible Fluid in the Rotating Coaxial Pipe. Vestnik Kibernetiki, 2017, no. 4 (28), pp. 56-61. (In Russian).

66. Kraiko A.N., Vatazhin A.B., Lyubimov G.A. Mekhanika zhidkosti i gaza. Izbrannoe [Fluid Mechanics. Selected, ed. by A.N. Kraiko]. Moscow, Fizmatlit Publ., 2013, 752 p. (In Russian).

67. Vatazhin A.B., Klimenko A.Yu., Lebedev A.B., Sorokin A.A. Homogeneous condens ation in turbulent submerged isobaric jets. Fluid Dynamics, 1988, vol. 23, iss. 2, pp. 194-203. DOI: $10.1007 /$ BF01051887.

68. Vasil'kov A.P. Calculation of a turbulent two-phase isobaric stream. Fluid Dynamics, 1976, vol. 11, iss. 5, pp. 699-704. DOI: 10.1007/BF01012960.

69. Shmiglevskiy Yu.D. On isobaric plane flows of a viscous incompressible fluid. USSR Computational Mathematics and Mathematical Physics, 1985, vol. 25, iss. 6, pp. 191-193. DOI: 10.1016/0041-5553(85)90030-8.

70. Shmyglevsky Yu. D. Analiticheskie issledovanija dinamiki gaza i zhidkosti. [Analytical Studies of Gas and Liquid Dynamics]. Moscow, 1999, 232 p. (In Russian).

71. Koterov V.N., Shmiglevskiy Yu.D., Scheprov A.V. A survey of analytical studies of steady viscous incompressible flows (2000-2004). Computational Mathematics and Mathematical Physics, 2005, vol. 45, no. 5, pp. 867-888.

72. Aristov S.N., Prosviryakov E.Y. Inhomogeneous Couette flow. Russian Journal of Nonlinear Dynamics, 2014, vol. 10, no. 2, pp. 177-182. DOI: 10.20537/nd1402004.

73. Aristov S.N., Prosviryakov E.Y. Stokes waves in vortical fluid. Rus. J. Nonlin. Dyn., 2014, vol. 10, no. 3, pp. 309-318. DOI: 10.20537/nd1403005.

74. Aristov S.N., Prosviryakov E.Y. Large-scale flows of viscous incompressible vortical fluid. Russian Aeronautics (IzVUZ), 2015, vol. 58, no. 4, pp. 413-418. DOI: 10.3103/S1068799815040091.

75. Aristov S.N., Prosviryakov E.Y. Unsteady layered vortical fluid flows. Fluid Dynamics, 2016, vol. 51, no. 2, pp. 148-154. DOI: 10.1134/S0015462816020034.

76. Aristov S.N., Knyazev D.V., Polyanin A.D. Exact solutions of the Navier-Stokes equations with the linear dependence of velocity components on two space variables. Theor. Found. Chem. Eng., 2009, vol. 43, no. 5, pp. 642-662. DOI: 10.1134/S0040579509050066.

77. Gershuni G.Z., Zhukhovitskii E.M. Instability of a system of horizontal layers of immiscible fluids heated from above. Fluid Dynamics, 1980, vol. 15, iss. 6, pp. 816-822. DOI: 10.1007/BF01096629.

78. Fikhtengolts G.M., Kurs differentsial'nogo i integral'nogo ischisleniya [A Course in Differential and Integral Calculus]. Moscow, Fizmatlit, 2001. (In Russian).

79. Kostrikin, A. I. Vvedenie v algebru. Chast I. Osnovy algebry: Uchebnik dlya vuzov [Introduction to algebra. Part 1. Bases of algebra: Textbook for institutions of higher learning]. Moscow, Fizmatlit, 2004, 272. (In Russian). 
Подана в журнал: 29.11.2018

УДК 539

DOI: $10.17804 / 2410-9908.2019 .1 .006-042$

\title{
ИССЛЕДОВАНИЕ ТЕМПЕРАТУРНОГО ПОЛЯ В СЛОИСТЫХ ТЕЧЕНИЯХ ВЕРТИКАЛЬНО ЗАВИХРЕННОЙ ВЯЗКОЙ НЕСЖИМАЕМОЙ ЖИДКОСТИ ПРИ ЗАДАНИИ ДВУХ ТЕРМОКАПИЛЛЯРНЫХ СИЛ НА СВОБОДНОЙ ГРАНИЦЕ
}

\author{
Н. В. Бурмашева ${ }^{\text {a)* }}$ Е. Ю. Просвиряков ${ }^{\text {) }}$ \\ Институт машиноведения УрО РАН, \\ ул. Комсомольская, 34, Екатеринбург, Российская Федеращия

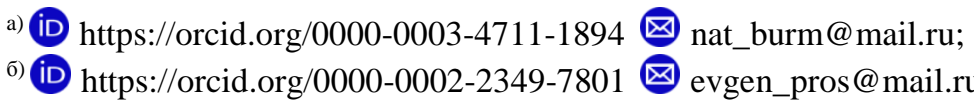 \\ *Ответственный автор. Электронная почта: nat_burm@mail.ru \\ Адрес для переписки: ул. Комсомольская, 34, Екатеринбург, Российская Федерация \\ Тел.: +7 (343) 375-35-76; факс: +7 (343) 374-53-30
}

В статье рассмотрено точное решение уравнений Обербека-Буссинеска, описывающее влияние термокапиллярного эффекта на конвективные течения вязкой несжимаемой жидкости. Класс точных решений выбран таким образом, что позволяет разрешить переопределенную систему уравнений движения жидкости за счет тождественного удовлетворения условию несжимаемости. В качестве граничных условий выбраны источники тепла на обеих границах, осуществляющие нагрев (охлаждение) жидкости. В статье получено точное решение и произведено исследование температурного поля. Показано, что структура точного решения не позволяет снизить размерность исследуемоймой краевой задачи, а также показано, что полученное точное решение допускает наличие противотечений в жидкости.

Ключевые слова: слоистое течение, точное решение, противотечения, конвекция Марангони, вертикальная закрутка, вихревое течение, система уравнений Обербека-Буссинеска.

\section{1. Введение}

Известно, что основой конвекции является движение молекул среды в противоположных направлениях под действием неравномерного нагревания/охлаждения этой среды. Само понятие конвекции было предложено для описания тепловых масс в нагретых движущихся жидкостях. На сегодняшний момент задача исследования особенностей конвективных движений является достаточно распространенной и встречается во многих теоретических и прикладных дисциплинах. Наиболее распространенным подходом для описания таких движений при нормальной гравитации является подход Обербека, подразумевающий линейную зависимость плотности жидкости от температуры. В результате объемные силы оказываются зависящими только от температуры [1]. Входящие в состав системы Обербека-Буссинеска уравнения Навье-Стокса обладают квадратичной нелинейностью, в результате чего порой даже в изотермическом случае их решения могут описывать появление застойных зон внутри жидкости.

Исторически первыми изотермическими точными решениями были решения Экмана [2] и Хименца [3], описывающие существование застойных точек и противотечений в потоке вязкой несжимаемой жидкости. Известно, что точное решение Экмана является основным инструментом для описания локализованных течений в океане и атмосфере. Модификации спиралевидного течения Экмана в горизонтальном бесконечном слое изотермической и неизотермической жидкости приведены в статьях, обзорах и монографиях [4-15]. 
Течение Хименца, описываемое нелинейной начально-краевой задачей для неустановившегося движения вязкой несжимаемой жидкости, является очень популярным объектом исследования в математической и теоретической гидродинамике. Стоит отметить, что в классической статье Хименца [3] рассматривались установившиеся течения. Нестационарные аналоги точного решения Хименца были исследованы Рябушинским в [16]. Многочисленные исследования по аналитическому и численному интегрированию течений типа Хименца-Рябушинского отражены в библиографических источниках [17-20]. Во всех публикациях [21-22], посвященных исследованию изотермических течений типа ХименцаРябушинского, при определенных значениях управляющих параметров краевых и начальнокраевых задач наблюдались противотоки в течении жидкости.

Исследования конвективных течений с помощью поля скоростей Хименца-Рябушинского показали, что влияние температурной стратификации может существенно изменить структуру поля скоростей [23-25]. В сравнении с изотермическим случаем в жидкости могут появиться дополнительные застойные точки и, как следствие, новые зоны с обратным (возвратным) течением [26-30]. В работах [31-38] представлены примеры краевых и начальнокраевых задач конвекции и термодиффузии с различными краевыми условиями, индуцирующими течение жидкости.

При моделировании крупномасштабных движений несжимаемых сред (течений в тонком слое) можно пренебрегать изменением поперечной скорости, полагая ее равной нулю [39]. В этом случае система уравнений Навье-Стокса становится переопределенной. Первым нетривиальным точным решением переопределенной системы уравнений Навье-Стокса является упоминавшееся уже течение Экмана [2]. Для невращающихся жидкостей при учете конвекции точными решениями для переопределенной системы уравнений Обербека-Буссинеска точными решениями является класс Остроумова-Бириха и его модификации и обобщения [40-52]. В статьях [53-64] рассмотрены приложения точного решения Остроумова-Бириха к вращающейся жидкости. Все течения, о которых шла речь выше, зависят от распределения давления.

Изобарические плоские течения вязкой несжимаемой жидкости (поле скоростей состоит из двух скоростей, которые зависят от двух координат и времени) начали изучать в [66]. В статьях [67-68] было построено много точных решений для изобарических течений. Систематическое исследование плоских течений жидкости было выполнено Шмыглевским [69-71].

Сравнительно недавно было показано существование точных решений для крупномасштабных сдвиговых течений вязкой несжимаемой жидкости [72-75]. Любопытно, что полученные решения описывают течения жидкости с вертикальной компонентой завихренности без учета вращения жидкости. В статье [76] показано, как изотермические точные решения могут быть использованы для исследования конвективных вихрей в вертикально завихренной жидкости. Решения, анонсированные в [31, 72-76], описывают конвективных течения типа Куэтта, индуцированные заданием скоростей и горизонтальных градиентов температуры на верхней границе бесконечного слоя жидкости. Основные обобщения течения Остроумова-Бириха были получены при учете термокапиллярных сил на межфазной недеформируемой границе. К настоящему времени не изучена конвекция Марангони для решений, представленных в [31], где предложено новое точное решение, описывающее движение вязкой несжимаемой вертикально завихренной жидкости под действием заданных на верхней границе термокапиллярных сил.

\section{2. Постановка задачи и методы решения}

Рассматривается течение жидкости в бесконечном горизонтальном слое толщиной $h$. При исследовании крупномасштабных движений в сдвиговых потоках вязкой несжимаемой жидкости (z-компонента $V_{z}$ вектора скорости $\boldsymbol{V}(x, y, z)$ полагается равной нулю) используется система уравнений тепловой конвекции в приближении Буссинеска: 


$$
\begin{gathered}
V_{x} \frac{\partial V_{x}}{\partial x}+V_{y} \frac{\partial V_{x}}{\partial y}=v \Delta V_{x}-\frac{\partial P}{\partial x} \\
V_{x} \frac{\partial V_{y}}{\partial x}+V_{y} \frac{\partial V_{y}}{\partial y}=v \Delta V_{y}-\frac{\partial P}{\partial y} \\
\frac{\partial P}{\partial z}=g \beta \Delta T \\
\frac{\partial T}{\partial x}+V_{y} \frac{\partial T}{\partial y}=\chi \Delta T \\
\frac{\partial V_{x}}{\partial x}+\frac{\partial V_{y}}{\partial y}=0 .
\end{gathered}
$$

Здесь введены обозначения: $V_{x}(x, y, z) ; V_{y}(x, y, z)$ - соответственно $x$ - и $y$ - компоненты вектора скорости $\boldsymbol{V}(x, y, z) ; P(x, y, z)$ - деленное на среднюю плотность отклонение давления от гидростатического; $T(x, y, z)$ - отклонение температуры от отсчетного значения; $v, \chi$ - кинематическая (молекулярная) вязкость жидкости и ее температуропроводность; $\Delta=\frac{\partial^{2}}{\partial x^{2}}+\frac{\partial^{2}}{\partial y^{2}}+\frac{\partial^{2}}{\partial z^{2}}-$ оператор Лапласа. Первые три уравнения системы (1) - это уравнения Навье-Стокса в проекциях на координатные оси, четвертое уравнение - это уравнение теплопроводности, последнее уравнение - это уравнение несжимаемости, в которых учтено условие $V_{z}=0$.

Особенностью системы (1) помимо ее нелинейности является ее переопределенность (число неизвестных меньше числа уравнений). Можно показать, что система (1) оказывается разрешимой в классе скоростей вида [57, 62]:

$$
V_{x}=U(z)+y u(z), V_{y}=V(z)
$$

поскольку при подстановке класса (2) в уравнение несжимаемости системы (1) последнее обращается в тождество.

Поля давления и температуры полагаем линейными формами относительно горизонтальных (продольных) $x, y[59,60]$ :

$$
T=T_{0}(z)+T_{1}(z) x+T_{2}(z) y, P=P_{0}(z)+P_{1}(z) x+P_{2}(z) y .
$$

Подстановка класса (2)-(3) в систему (1) при использовании метода неопределенных коэффициентов приводит последнюю к системе обыкновенных дифференциальных уравнений

$$
\begin{gathered}
u^{\prime \prime}(z)=0, T_{1}{ }^{\prime}(z)=0, P_{1}^{\prime}(z)=g \beta T_{1}(z), \chi T_{2}{ }^{\prime}(z)=u(z) T_{1}(z) ; \\
P_{2}^{\prime}(z)=g \beta T_{2}(z), v V^{\prime \prime}(z)=P_{2}(z), v U^{\prime \prime}(z)=V(z) u(z)+P_{1}(z) ; \\
\chi T_{0}{ }^{\prime \prime}(z)=U(z) T_{1}(z)+V(z) T_{2}(z), P_{0}^{\prime}(z)=g \beta T_{0}(z) .
\end{gathered}
$$


Здесь и далее штрихом обозначена полная производная по вертикальной координате $z$. Уравнения системы (4) интегрируются в том порядке, в котором они приведены. Среди уравнений системы (4) только первые два уравнения являются изолированными (т. е. интегрируются независимо от других), остальные уравнения являются пассивными (т. е. их решения следует искать только после того, как найдены решения изолированных уравнений). Общее решение системы (4), полученное последовательным интегрированием входящих в состав системы уравнений, имеет вид:

$$
\begin{aligned}
& u=C_{1} z+C_{5} \\
& T_{1}=C_{2} z+C_{3} \\
& P_{1}=g \beta\left(\frac{1}{2} z^{2} C_{2}+z C_{3}\right)+C_{4} \\
& T_{2}=\frac{\frac{1}{2} z^{4} C_{1} C_{2}+3 z^{2} C_{3} C_{5}+z^{3}\left(C_{1} C_{3}+C_{2} C_{5}\right)+6 z \chi C_{6}}{6 \chi}+C_{7} ; \\
& P_{2}=\frac{g \beta\left(\frac{1}{5} z^{5} C_{1} C_{2}+2 z^{3} C_{3} C_{5}+\frac{1}{2} z^{4}\left(C_{1} C_{3}+C_{2} C_{5}\right)+6 z^{2} \chi C_{6}+12 z \chi C_{7}\right)}{12 \chi}+C_{8} \\
& V=\frac{\frac{1}{7} g z^{7} \beta C_{1} C_{2}+3 g z^{5} \beta C_{3} C_{5}+\frac{1}{2} g z^{6} \beta\left(C_{1} C_{3}+C_{2} C_{5}\right)}{360 v \chi}+ \\
& +\frac{g z^{4} \beta \chi C_{6}+4 g z^{3} \beta \chi C_{7}+12 z^{2} \chi C_{8}+24 z v \chi C_{9}}{24 v \chi}+C_{10} \\
& U=\frac{1}{362880 v^{2} \chi}\left[15120 g z^{4} \beta v \chi C_{2}+\frac{8}{5} g z^{10} \beta C_{1}^{2} C_{2}+60480 g z^{3} \beta v \chi C_{3}+\right. \\
& +9 g z^{8} \beta C_{5}\left(7 C_{1} C_{3}+C_{2} C_{5}\right)+g z^{9} \beta C_{1}\left(7 C_{1} C_{3}+9 C_{2} C_{5}\right)+72 g z^{7} \beta\left(C_{3} C_{5}^{2}+5 \chi C_{1} C_{6}\right)+3024 g z^{5} \beta \chi C_{5} C_{7}+ \\
& +504 g z^{6} \beta \chi\left(C_{5} C_{6}+4 C_{1} C_{7}\right)+9072 z^{5} \chi C_{1} C_{8}+15120 z^{4} \chi\left(C_{5} C_{8}+2 v C_{1} C_{9}\right)+ \\
& \left.+60480 z^{3} v \chi\left(C_{5} C_{9}+C_{1} C_{10}\right)+181440 z^{2} v \chi\left(C_{4}+C_{5} C_{10}\right)+362880 z v^{2} \chi C_{11}\right]+C_{12} \\
& T_{0}=\frac{1}{119750400 v^{2} \chi^{3}}\left[\frac{22}{13} g z^{13} \beta(15 v+2 \chi) C_{1}^{2} C_{2}^{2}+\frac{1}{2} g z^{12} \beta C_{1} C_{2}\left((330 v+43 \chi) C_{1} C_{3}+\right.\right. \\
& \left.+(330 v+45 \chi) C_{2} C_{5}\right)+3 g z^{11} \beta\left(7(12 v+\chi) C_{1}^{2} C_{3}^{2}+(492 v+72 \chi) C_{1} C_{2} C_{3} C_{5}+(84 v+9 \chi) C_{2}^{2} C_{5}^{2}\right)+ \\
& +33 g z^{10} \beta\left((84 v+7 \chi) C_{1} C_{3}^{2} C_{5}+(84 v+9 \chi) C_{2} C_{3} C_{5}^{2}+(156 v+40 \chi) \chi C_{1} C_{2} C_{6}\right)+
\end{aligned}
$$




$$
\begin{gathered}
+330 g z^{9} \beta\left((21 v+\chi) C_{3}^{2} C_{5}^{2}+(42 v+5 \chi) \chi C_{1} C_{3} C_{6}+(42 v+7 \chi) \chi C_{2} C_{5} C_{6}+(72 v+28 \chi) \chi C_{1} C_{2} C_{7}\right)+ \\
+2970 g z^{8} \beta \chi\left((21 v+\chi) C_{3} C_{5} C_{6}+(21 v+4 \chi) C_{1} C_{3} C_{7}+(21 v+6 \chi) C_{2} C_{5} C_{7}\right)+ \\
+23760 g z^{7} \beta \chi\left(5 v \chi C_{2}^{2}+5 v \chi C_{6}^{2}+(11 v+\chi) C_{3} C_{5} C_{7}\right)+
\end{gathered}
$$$$
+831600 g z^{6} \beta v \chi^{2}\left(C_{2} C_{3}+C_{6} C_{7}\right)+997920 g z^{5} \beta v \chi^{2}\left(C_{3}^{2}+C_{7}^{2}\right)+17820 z^{8} \chi(5 v+3 \chi) C_{1} C_{2} C_{8}+
$$$$
+23760 z^{7} \chi\left((10 v+3 \chi) C_{1} C_{3} C_{8}+(10 v+5 \chi) C_{2} C_{5} C_{8}+10 v(v+\chi) C_{1} C_{2} C_{9}\right)+
$$$$
+166320 z^{6} \chi\left((6 v+\chi) C_{3} C_{5} C_{8}+2 v(2 v+\chi) C_{1} C_{3} C_{9}+4 v(v+\chi) C_{2} C_{5} C_{9}+2 v(v+2 \chi) C_{1} C_{2} C_{10}\right)+
$$$$
+997920 z^{5} v \chi\left(3 \chi C_{2} C_{4}+3 \chi C_{6} C_{8}+(3 v+\chi) C_{3} C_{5} C_{9}+(v+\chi) C_{1} C_{3} C_{10}+(v+3 \chi) C_{2} C_{5} C_{10}\right)+
$$$$
+4989600 z^{4} v \chi\left(\chi C_{3} C_{4}+\chi C_{7} C_{8}+2 v \chi C_{6} C_{9}+(v+\chi) C_{3} C_{5} C_{10}+2 v \chi C_{2} C_{11}\right)+
$$$$
+19958400 z^{3} v^{2} \chi^{2}\left(C_{7} C_{9}+C_{6} C_{10}+C_{3} C_{11}+C_{2} C_{12}\right)+
$$$$
\left.+59875200 z^{2} v^{2} \chi^{2}\left(C_{7} C_{10}+C_{3} C_{12}\right)+119750400 z v^{2} \chi^{3} C_{13}\right]+C_{14}
$$$$
P_{0}=\frac{g \beta}{3113510400 v^{2} \chi^{3}}\left[\frac{22}{7} g z^{14} \beta(15 v+2 \chi) C_{1}^{2} C_{2}^{2}+\right.
$$$$
+g z^{13} \beta C_{1} C_{2}\left((330 v+43 \chi) C_{1} C_{3}+(330 v+45 \chi) C_{2} C_{5}\right)+
$$$$
+\frac{13}{2} g z^{12} \beta\left((4 v+7 \chi) C_{1}^{2} C_{3}^{2}+(492 v+72 \chi) C_{1} C_{2} C_{3} C_{5}+(84 v+9 \chi) C_{2}^{2} C_{5}^{2}\right)+
$$$$
+78 g z^{11} \beta\left((84 v+7 \chi) C_{1} C_{3}^{2} C_{5}+(84 v+9 \chi) C_{2} C_{3} C_{5}^{2}+(156 v+40 \chi) C_{1} C_{2} C_{6}\right)+
$$$$
+858 g z^{10} \beta\left((21 v+\chi) C_{3}^{2} C_{5}^{2}+(42 v+5 \chi) \chi C_{1} C_{3} C_{6}+\right.
$$$$
\left.+(42 v+7 \chi) \chi C_{2} C_{5} C_{6}+(72 v+28 \chi) \chi C_{1} C_{2} C_{7}\right)+
$$$$
+8580 g z^{9} \beta \chi\left((21 v+\chi) C_{3} C_{5} C_{6}+(21 v+4 \chi) C_{1} C_{3} C_{7}+(21 v+6 \chi) C_{2} C_{5} C_{7}\right)+
$$

$+3088800 g z^{7} \beta v \chi^{2}\left(C_{2} C_{3}+C_{6} C_{7}\right)+77220 g z^{8} \beta \chi\left(5 v \chi C_{2}^{2}+5 v \chi C_{6}^{2}+(11 v+\chi) C_{3} C_{5} C_{7}\right)+$

$$
+4324320 g z^{6} \beta v \chi^{2}\left(C_{3}^{2}+C_{7}^{2}\right)+51480 z^{9} \chi(5 v+3 \chi) C_{1} C_{2} C_{8}+
$$

Burmasheva N. V., Prosviryakov E. Yu. Temperature field investigation in layered flows of a vertically swirling viscous incompressible fluid under two thermocapillar forces at a free boundary // Diagnostics, Resource and Mechanics of materials and structures. - 2019. - Iss. 1. - P. 6-42. - DOI: 10.17804/2410-9908.2019.1.006-042. 


$$
\begin{gathered}
+77220 z^{8} \chi\left((10 v+3 \chi) C_{1} C_{3} C_{8}+(10 v+5 \chi) C_{2} C_{5} C_{8}+10 v(v+\chi) C_{1} C_{2} C_{9}\right)+ \\
+617760 z^{7} \chi\left((6 v+\chi) C_{3} C_{5} C_{8}+2 v(2 v+\chi) C_{1} C_{3} C_{9}+4 v(v+\chi) C_{2} C_{5} C_{9}+2 v(v+2 \chi) C_{1} C_{2} C_{10}\right)+ \\
+4324320 z^{6} v \chi\left(3 \chi C_{2} C_{4}+3 \chi C_{6} C_{8}+(3 v+\chi) C_{3} C_{5} C_{9}+(v+\chi) C_{1} C_{3} C_{10}+(v+3 \chi) C_{2} C_{5} C_{10}\right)+ \\
+25945920 z^{5} v \chi\left(\chi C_{3} C_{4}+\chi C_{7} C_{8}+2 v \chi C_{6} C_{9}+(v+\chi) C_{3} C_{5} C_{10}+2 v \chi C_{2} C_{11}\right)+ \\
+129729600 z^{4} v^{2} \chi^{2}\left(C_{7} C_{9}+C_{6} C_{10}+C_{3} C_{11}+C_{2} C_{12}\right)+ \\
\left.+518918400 z^{3} v^{2} \chi^{2}\left(C_{7} C_{10}+C_{3} C_{12}\right)+1556755200 z^{2} v^{2} \chi^{3} C_{13}+3113510400 z v^{2} \chi^{3} C_{14}\right]+C_{15} .
\end{gathered}
$$

Для определения возникших в ходе интегрирования системы (4) констант $C_{i}$, где $i=1, \ldots, 15$, необходимо задать соответствующее число граничных условий. Будем полагать, что нижняя поверхность $z=0$ рассматриваемого бесконечного горизонтального слоя является абсолютно твердой, верхняя поверхность $z=h-$ свободной и недеформируемой [77]. Рассмотрим далее краевую задачу, описывающую термокапиллярный эффект на верхней границе слоя, т. е. на верхней границе слоя действуют термокапиллярные силы, генерирующие конвективные течения вертикально завихренной жидкости:

$$
\eta V_{x}^{\prime}(h)=-\sigma \frac{\partial T}{\partial x}(h), \eta V_{y}^{\prime}(h)=-\sigma \frac{\partial T}{\partial y}(h)
$$

На обеих границах заданы источники тепла

$$
T(x, y, 0)=A x+B y, T(x, y, h)=\vartheta+C x+D y .
$$

Здесь $A, B$ и $C, D$ - значения продольных градиентов температуры, принимаемые на нижней и верхней границах рассматриваемого бесконечного слоя соответственно, $\vartheta-$ значение температуры на свободной границе.

При этом нижнюю границу $z=0$ считаем отсчетным уровнем температуры, аналогичным образом верхнюю границу $z=h$ - отсчетным уровнем давления:

$$
P(x, y, h)=0 \text {. }
$$

Также полагаем, что нижняя граница движется со скоростью, определяемой выражениями

$$
V_{x}=W \cos \alpha+\Omega y ; V_{y}=W \sin \alpha ;
$$

где $\Omega$ - значение горизонтального градиента скорости $V_{x}$ на нижней границе (пространственное ускорение); $W$ - модуль однородной составляющей скорости на неподвижной границе слоя жидкости. Получим

Подставим выбранный класс решений (2)-(3) в первое обозначенное краевое условие. 


$$
\eta V_{x}^{\prime}(h)=-\sigma \frac{\partial T}{\partial x}(h)
$$

здесь $\sigma$ - температурный коэффициент поверхностного натяжения.

Подставим в это граничное условие скорости (2):

$$
\left.\eta \frac{\partial}{\partial z}(U(z)+y u(z))\right|_{z=h}=-\left.\sigma \frac{\partial}{\partial x}\left(T_{0}(z)+x T_{1}(z)+y T_{2}(z)\right)\right|_{z=h},
$$

откуда в силу метода неопределенных коэффициентов следуют условия

$$
\eta U^{\prime}(h)=-\sigma T_{1}(h) ; u^{\prime}(h)=0 \text {. }
$$

Аналогичным образом получаем

$$
\eta V^{\prime}(h)=-\sigma T_{2}(h)
$$

С учетом структуры класса (2)-(3) окончательно приходим к системе из пятнадцати краевых условий:

$$
\begin{gathered}
u(0)=\Omega, u^{\prime}(h)=0 ; \\
T_{0}(0)=0, T_{1}(0)=A, T_{2}(0)=B \\
T_{0}(h)=\vartheta, T_{1}(h)=C, T_{2}(h)=D \\
P_{0}(h)=0, P_{1}(h)=0, P_{2}(h)=0 \\
U(0)=W \cos \alpha, V(0)=W \sin \alpha \\
\eta U^{\prime}(h)=-\sigma T_{1}(h), \eta V^{\prime}(h)=-\sigma T_{2}(h) .
\end{gathered}
$$

Формулы (5) характеризуют неоднородное распределение скоростей на нижней границе. Отметим, что выбор граничных условий (5) обусловлен масштабами изучаемого течения.

Рассмотрим далее частный случай нагрева (охлаждения) верхней границы $(A=B=0)$ и проанализируем поведение температурного поля. Решения большинства уравнений системы (4) зависят от градиента температуры $T_{1}$, а следовательно и от принимаемых им на границах слоя значений. Различие этих значений ведет к большему разнообразию свойств решений остальных рассматриваемых в задаче полей. При задании двух в общем случае различных тепловых источников на верхней границе получаем две отличные друг от друга термокапиллярные силы, которые в свою очередь индуцируют потоки, оказывающие различное влияние на формирование результирующего течения. 


\section{3. Результаты и обсуждение}

\section{1. Точное решение краевой задачи}

Найдем, используя систему ограничений (5), точное решение поставленной краевой задачи (4)-(5). Начнем с продольного градиента скорости $u$. Подставляя в найденное решение

$$
u=C_{1} z+C_{5}
$$

значения аргумента $z=0$ и $z=h$, приходим к системе

$$
\left\{\begin{array}{l}
C_{5}=\Omega, \\
C_{1}=0,
\end{array}\right.
$$

откуда

$$
u=\Omega \text {. }
$$

Стоит отметить, что если при рассмотрении обобщенных классов для поля температуры, нелинейно зависящей от продольных координат, градиент скорости $u$ мог бы линейно зависеть от поперечной координаты $z$ даже при аналогичном задании термокапиллярных сил на верхней границе.

Далее переходим к продольному градиенту температуры

$$
T_{1}=\tilde{N}_{2} z+C_{3} .
$$

Значения констант интегрирования $C_{2}, C_{3}$ определяются из граничных условий:

$$
A=T_{1}(0)=C_{2} \cdot 0+C_{3} ; \tilde{N}=T_{1}(h)=\tilde{N}_{2} \cdot h+C_{3} .
$$

Разрешая эту систему, легко находим

$$
C_{3}=A ; \tilde{N}_{2}=\frac{C-A}{h} .
$$

А значит, окончательно функция $T_{1}$ принимает вид:

$$
T_{1}=A+\frac{C-A}{h} z
$$

Подставляя найденные значения констант $C_{2}, C_{3}$ и пользуясь соответствующим граничным условием для давления, находим константу

$$
C_{4}=-\frac{g \beta h(A+C)}{2},
$$

входящую в выражение для градиента давления $P_{1}$, в силу чего $P_{1}$ принимает вид:

$$
P_{1}=\frac{g \beta}{2 h}\left((C-A) z^{2}+2 A h z-(A+C) h^{2}\right)
$$

Burmasheva N. V., Prosviryakov E. Yu. Temperature field investigation in layered flows of a vertically swirling viscous incompressible fluid under two thermocapillar forces at a free boundary // Diagnostics, Resource and Mechanics of materials and structures. - 2019. - Iss. 1. - P. 6-42. - DOI: 10.17804/2410-9908.2019.1.006-042. 
Интегрируя аналогичным образом остальные уравнения системы (4), получаем точное решение краевой задачи (4)-(5):

$$
\begin{aligned}
& u=\Omega \text {; } \\
& T_{1}=A+\frac{C-A}{h} z \\
& P_{1}=\frac{g \beta}{2 h}\left((C-A) z^{2}+2 A h z-(A+C) h^{2}\right) \\
& T_{2}=B+\frac{D-B}{h} z+\frac{\Omega z}{6 h \chi}\left((C-A) z^{2}+3 A h z-(2 A+C) h^{2}\right) \\
& P_{2}=\frac{g \beta}{2 h}\left((D-B) z^{2}+2 B h z-(B+D) h^{2}\right)+\frac{g \beta \Omega}{24 h \chi}\left((C-A) z^{4}+4 A h z^{3}-2(2 A+C) h^{2} z^{2}+(A+C) h^{4}\right) \\
& V=W \sin \alpha-\frac{D \sigma}{\eta} z+\frac{g \beta z}{24 h v}\left((D-B) z^{3}+4 B h z^{2}-6(B+D) h^{2} z+4(B+2 D) h^{3}\right)- \\
& -\frac{g \beta \Omega}{720 h v \chi} z\left((C-A) z^{5}-6 A h z^{4}+5(2 A+C) h^{2} z^{3}-15(A+C) h^{4} z+2(7 A+8 C) h^{5}\right) \\
& U=W \cos \alpha-\frac{C \sigma}{\eta} z+\frac{g \beta}{24 h v} z\left((C-A) z^{3}+4 A h z^{2}-6(A+C) h^{2} z+4(A+2 C) h^{3}\right)- \\
& -\frac{g \beta \Omega}{720 h v^{2}} z\left((D-B) z^{5}-6 B h z^{4}+15(B+D) h^{2} z^{3}-20(B+2 D) h^{3} z^{2}+6(4 B+11 D) h^{5}\right)- \\
& -\frac{W \sin \alpha \Omega}{2 v}(2 h-z) z+\frac{D \sigma \Omega}{6 \eta v}\left(3 h^{2}-z^{2}\right) z+\frac{g \beta \Omega^{2} z}{120960 h v^{2} \chi}\left[3(-A+C) z^{7}+24 A h z^{6}-28(2 A+C) h^{2} z^{5}+\right. \\
& \left.+210(A+C) h^{4} z^{3}-(392 A+448 C) h^{5} z^{2}+(528 A+648 C) h^{7}\right] \text {; } \\
& T_{0}=\frac{z \vartheta}{h}-\frac{g \beta}{5040 h^{2} v \chi}(h-z) z\left[5\left(A^{2}+B^{2}-2 A C+C^{2}-2 B D+D^{2}\right) z^{5}+\right. \\
& +5\left(-6 A^{2}-6 B^{2}+5 A C+C^{2}+5 B D+D^{2}\right) h z^{4}++\left(75 A^{2}+75 B^{2}+25 A C-58 C^{2}+25 B D-58 D^{2}\right) h^{2} z^{3}+ \\
& +\left(-100 A^{2}-100 B^{2}-150 A C+82 C^{2}-150 B D+82 D^{2}\right) h^{3} z^{2}+ \\
& +\left(40 A^{2}+40 B^{2}+130 A C+82 C^{2}+130 B D+82 D^{2}\right) h^{4} z+ \\
& \left.+\left(40 A^{2}+40 B^{2}+130 A C+82 C^{2}+130 B D+82 D^{2}\right) h^{5}\right]-
\end{aligned}
$$

Burmasheva N. V., Prosviryakov E. Yu. Temperature field investigation in layered flows of a vertically swirling viscous incompressible fluid under two thermocapillar forces at a free boundary // Diagnostics, Resource and Mechanics of materials and structures. - 2019. - Iss. 1. - P. 6-42. - DOI: 10.17804/2410-9908.2019.1.006-042. 


$$
\begin{aligned}
& -\frac{W z}{6 h \chi}\left((2 B+D) h^{2} \sin \alpha-3(A \cos \alpha+B \sin \alpha) h z+((A-C) \cos \alpha+(B-D) \sin \alpha) z^{2}\right)+ \\
& +\frac{\sigma(h-z) z}{12 h \eta \chi}\left(\left(-A C+C^{2}-B D+D^{2}\right) z^{2}+\left(A C+C^{2}+B D+D^{2}\right) h z+\left(A C+C^{2}+B D+D^{2}\right) h^{2}\right)+ \\
& +\frac{g \beta \Omega(h-z) z}{120960 h^{2} v \chi^{2}}\left[14(-A B+B C+A D-C D) z^{7}+(112 A B-49 B C-49 A D-14 C D) h z^{6}+\right. \\
& +(-352 A B+31 B C-89 A D+146 C D) h^{2} z^{5}+(554 A B+59 B C+639 A D-78 C D) h^{3} z^{4}- \\
& -(338 A B+67 B C+999 A D+456 C D) h^{4} z^{3}+(-184 A B-221 B C+317 A D+328 C D) h^{5} z^{2}+ \\
& \left.+(208 A B+227 B C+317 A D+328 C D) h^{6} z^{3}+(208 A B+227 B C+317 A D+328 C D) h^{7}\right]+ \\
& +\frac{W \sin \alpha \Omega(h-z) z}{360 h \chi^{2}}\left(3(A-C) z^{3}-3(4 A+C) h z^{2}+(8 A+7 C) h^{2} z+(8 A+7 C) h^{3}\right)- \\
& -\frac{D \sigma \Omega(h-z) z}{360 h \eta \chi^{2}}\left(2(A-C) z^{4}-(7 A+2 C) h z^{3}+3(A+C) h^{2} z^{2}+3(A+C) h^{3} z+3(A+C) h^{4}\right)+ \\
& +\frac{g \beta \Omega(h-z) z}{362880 h^{2} v^{2} \chi}\left[7(-A B+B C+A D-C D) z^{7}+(56 A B-47 B C-2 A D-7 C D) h z^{6}+\right. \\
& +(-196 A B+133 B C-182 A D+173 C D) h^{2} z^{5}+(392 A B-203 B C+742 A D-499 C D) h^{3} z^{4}- \\
& -(112 A B+203 B C+266 A D+499 C D) h^{4} z^{3}+(-1120 A B+805 B C-3038 A D+2273 C D) h^{5} z^{2}+ \\
& \left.+(896 A B+805 B C+2506 A D+2273 C D) h^{6} z+(896 A B+805 B C+2506 A D+2273 C D) h^{7}\right]+ \\
& +\frac{W \sin \alpha \Omega(h-z) z}{120 h v \chi}\left(3(A-C) z^{3}+(-12 A+7 C) h z^{2}+(8 A+7 C) h^{2} z+(8 A+7 C) h^{3}\right)-
\end{aligned}
$$$$
-\frac{D \sigma \Omega(h-z) z}{360 h \eta v \chi}\left(2(A-C) z^{4}-(A+2 C) h z^{3}+(-16 A+13 C) h^{2} z^{2}+(14 A+13 C) h^{3} z+(14 A+13 C) h^{4}\right)-
$$$$
-\frac{g \beta \Omega^{2}(h-z) z}{33916800 h^{2} v \chi^{3}}\left[84\left(A^{2}-2 A C+C^{2}\right) z^{9}+\left(-840 A^{2}+756 A C+84 C^{2}\right) h z^{8}+\right.
$$$$
+\left(3010 A^{2}-14 A C-686 C^{2}\right) h^{2} z^{7}-\left(3920 A^{2}+3479 A C+686 C^{2}\right) h^{3} z^{6}+
$$$$
+\left(-2820 A^{2}+921 A C+3714 C^{2}\right) h^{4} z^{5}+\left(15352 A^{2}+15397 A C-1214 C^{2}\right) h^{5} z^{4}-
$$$$
-\left(17912 A^{2}+27569 A C+8144 C^{2}\right) h^{6} z^{3}+\left(3648 A^{2}+7851 A C+4176 C^{2}\right) h^{7} z^{2}+
$$

Burmasheva N. V., Prosviryakov E. Yu. Temperature field investigation in layered flows of a vertically swirling viscous incompressible fluid under two thermocapillar forces at a free boundary // Diagnostics, Resource and Mechanics of materials and structures. - 2019. - Iss. 1. - P. 6-42. - DOI: 10.17804/2410-9908.2019.1.006-042. 


$$
\begin{aligned}
& \left.+\left(3648 A^{2}+7851 A C+4176 C^{2}\right) h^{8} z+\left(3648 A^{2}+7851 A C+4176 C^{2}\right) h^{9}\right]- \\
& -\frac{g \beta \Omega^{2}(h-z) z}{119750400 h^{2} v^{2} \chi^{2}}\left[27\left(A^{2}-2 A C+C^{2}\right) z^{9}+\left(-270 A^{2}+243 A C+27 C^{2}\right) h z^{8}+\right. \\
& +\left(830 A^{2}-142 A C-358 C^{2}\right) h^{2} z^{7}-\left(160 A^{2}+637 A C+358 C^{2}\right) h^{3} z^{6}+ \\
& +\left(-5110 A^{2}-637 A C+4592 C^{2}\right) h^{4} z^{5}+\left(14756 A^{2}+8141 A C-10192 C^{2}\right) h^{5} z^{4}- \\
& -\left(4648 A^{2}+14035 A C+10192 C^{2}\right) h^{6} z^{3}+\left(-48208 A^{2}-23935 A C+43268 C^{2}\right) h^{7} z^{2}+ \\
& \left.+\left(38912 A^{2}+82985 A C+43268 C^{2}\right) h^{8} z+\left(38912 A^{2}+82985 A C+43268 C^{2}\right) h^{9}\right], \\
& P_{0}=\frac{g^{2} \beta^{2}(h-z)^{2}}{40320 h^{2} v \chi}\left[5\left(A^{2}+B^{2}-2 A C+C^{2}-2 B D+D^{2}\right) z^{6}+10\left(-3 A^{2}-3 B^{2}+2 A C+C^{2}+2 B D+D^{2}\right) h z^{5}+\right. \\
& +\left(75 A^{2}+75 B^{2}+50 A C-69 C^{2}+50 B D-69 D^{2}\right) h^{2} z^{4}+ \\
& +4\left(-25 A^{2}-25 B^{2}-50 A C+19 C^{2}-50 B D+19 D^{2}\right) h^{3} z^{3}+ \\
& +\left(5 A^{2}+5 B^{2}+110 A C+221 C^{2}+110 B D+221 D^{2}\right) h^{4} z^{2}+ \\
& +\left(110 A^{2}+110 B^{2}+420 A C+336 C^{2}+420 B D+336 D^{2}\right) h^{5} z+ \\
& \left.+\left(55 A^{2}+55 B^{2}+210 A C+183 C^{2}+210 B D+183 D^{2}\right) h^{6}\right]-\frac{g \beta \vartheta(h-z)(h+z)}{2 h}+ \\
& +\frac{W g \beta(h-z)^{2}}{24 h \chi}\left[((-A+C) \cos \alpha+(-B+D) \sin \alpha) z^{2}+2((A+C) \cos \alpha+(B+D) \sin \alpha) h z+\right. \\
& \left.+((A+C) \cos \alpha+(B+D) \sin \alpha) h^{2}\right]-\frac{g \beta \sigma(h-z)^{2}}{120 h \eta \chi}\left[\left(-2 A C+2 C^{2}-2 B D+2 D^{2}\right) z^{3}+\right. \\
& \left.+\left(A C+4 C^{2}+B D+4 D^{2}\right) h z^{2}+2\left(2 A C+3 C^{2}+2 B D+3 D^{2}\right) h^{2} z+\left(2 A C+3 C^{2}+2 B D+3 D^{2}\right) h^{3}\right]- \\
& -\frac{g^{2} \beta^{2} \Omega(h-z)^{2}}{1209600 h^{2} v \chi^{2}}\left[14(-A B+B C+A D-C D) z^{8}+(112 A B-42 B C-42 A D-28 C D) h z^{7}+\right. \\
& +(-342 A B+2 B C-148 A D+158 C D) h^{2} z^{6}+(484 A B+86 B C+786 A D+24 C D) h^{3} z^{5}- \\
& -10(16 A B+4 B C+101 A D+74 C D) h^{4} z^{4}-(496 A B+474 B C+174 A D-64 C D) h^{5} z^{3}+
\end{aligned}
$$

Burmasheva N. V., Prosviryakov E. Yu. Temperature field investigation in layered flows of a vertically swirling viscous incompressible fluid under two thermocapillar forces at a free boundary // Diagnostics, Resource and Mechanics of materials and structures. - 2019. - Iss. 1. - P. 6-42. - DOI: 10.17804/2410-9908.2019.1.006-042. 


$$
\begin{aligned}
& +(148 A B+212 B C+662 A D+868 C D) h^{6} z^{2}+(792 A B+898 B C+1498 A D+1672 C D) h^{7} z+ \\
& \left.+(396 A B+449 B C+749 A D+836 C D) h^{8}\right]-\frac{g \beta W \sin \alpha \Omega(h-z)^{2}}{720 h \chi^{2}}\left[4(A-C) z^{4}-2(2 A+C) h z^{3}+\right. \\
& \left.+(A+2 C) h^{2} z^{2}+6(A+C) h^{3} z+3(A+C) h^{4}\right]+\frac{g \beta D \sigma \Omega(h-z)^{2}}{5040 h \eta \chi^{2}}\left[4(A-C) z^{5}-(13 A+8 C) h z^{4}+\right. \\
& \left.+2(-A+C) h^{2} z^{3}+(9 A+12 C) h^{3} z^{2}+2(10 A+11 C) h^{4} z+(10 A+11 C) h^{5}\right]- \\
& -\frac{g^{2} \beta^{2} \Omega(h-z)^{2}}{3628800 h^{2} v^{2} \chi}\left[7(-A B+B C+A D-C D) z^{8}+(56 A B-46 B C+4 A D-14 C D) h z^{7}+\right. \\
& +(-196 A B+126 B C-224 A D+204 C D) h^{2} z^{6}+(392 A B-182 B C+868 A D-538 C D) h^{3} z^{5}+ \\
& +(140 A B-490 B C 280 A D-1280 C D) h^{4} z^{4}+(-2128 A B+1218 B C-5852 A D+3522 C D) h^{5} z^{3}+ \\
& +(644 A B+2926 B C+1876 A D+8324 C D) h^{6} z^{2}+(3416 A B+4634 B C+9604 A D+13126 C D) h^{7} z+ \\
& \left.+(1708 A B+2317 B C+4802 A D+6563 C D) h^{8}\right]-\frac{g \beta W \sin \alpha \Omega(h-z)^{2}}{240 h v \chi}\left[(A-C) z^{4}+\right. \\
& \left.+2(-2 A+C) h z^{3}+(A+5 C) h^{2} z^{2}+2(3 A+4 C) h^{3} z+(3 A+4 C) h^{4}\right]+ \\
& +\frac{g \beta D \sigma \Omega(h-z)^{2}}{5040 h \eta v \chi}\left[4(A-C) z^{5}+(A-8 C) h z^{4}+2(-22 A+15 C) h^{2} z^{3}+4(4 A+17 C) h^{3} z^{2}+\right. \\
& \left.+2(38 A+53 C) h^{4} z+(38 A+53 C) h^{5}\right]+\frac{g^{2} \beta^{2} \Omega^{2}(h-z)^{2}}{79833600 h^{2} v \chi^{3}}\left[14\left(A^{2}-2 A C+C^{2}\right) z^{10}+\right. \\
& +\left(-140 A^{2}+112 A C+28 C^{2}\right) h z^{9}+\left(476 A^{2}+98 A C-112 C^{2}\right) h^{2} z^{8}-\left(448 A^{2}+686 A C+252 C^{2}\right) h^{3} z^{7}+ \\
& +\left(-1097 A^{2}-370 A C+708 C^{2}\right) h^{4} z^{6}+\left(3446 A^{2}+4082 A C+260 C^{2}\right) h^{5} z^{5}- \\
& -\left(3099 A^{2}+5788 A C+2498 C^{2}\right) h^{6} z^{4}-\left(1020 A^{2}+1490 A C+328 C^{2}\right) h^{7} z^{3}+ \\
& +\left(1059 A^{2}+2808 A C+1842 C^{2}\right) h^{8} z^{2}+\left(3138 A^{2}+7106 C+4012 C^{2}\right) h^{9} z+ \\
& \left.+\left(1569 A^{2}+3553 A C+2006 C^{2}\right) h^{10}\right]+ \\
& +\frac{g^{2} \beta^{2} \Omega^{2}(h-z)^{2}}{479001600 h^{2} v^{2} \chi^{2}}\left[9\left(A^{2}-2 A C+C^{2}\right) z^{10}+9\left(-10 A^{2}+8 A C+2 C^{2}\right) h z^{9}+\right.
\end{aligned}
$$

Burmasheva N. V., Prosviryakov E. Yu. Temperature field investigation in layered flows of a vertically swirling viscous incompressible fluid under two thermocapillar forces at a free boundary // Diagnostics, Resource and Mechanics of materials and structures. - 2019. - Iss. 1. - P. 6-42. - DOI: 10.17804/2410-9908.2019.1.006-042. 


$$
\begin{gathered}
+\left(251 A^{2}+8 A C-127 C^{2}\right) h^{2} z^{8}+\left(152 A^{2}-276 A C-272 C^{2}\right) h^{3} z^{7}+ \\
+\left(-2422 A^{2}-560 A C+2058 C^{2}\right) h^{4} z^{6}+\left(6356 A^{2} 4172 A C-4060 C^{2}\right) h^{5} z^{5}+ \\
+\left(2198 A^{2}-5880 A C-10178 C^{2}\right) h^{6} z^{4}+\left(-36808 A^{2}-23852 A C+26472 C^{2}\right) h^{7} z^{3}+ \\
+\left(11306 A^{2}+65096 A C+63122 C^{2}\right) h^{8} z^{2}+\left(59420 A^{2}+154044 A C+99772 C^{2}\right) h^{9} z+ \\
\left.+\left(29710 A^{2}+77022 A C+49886 C^{2}\right) h^{10}\right] .
\end{gathered}
$$

\section{2. Случай нагрева верхней границы}

Проанализируем приведенное выше точное решение (6) в частном случае задания источников тепла только на верхней границе слоя. Для этого необходимо положить $A=B=0$, в результате чего общее решение (6) принимает вид:

$$
\begin{aligned}
& u=\Omega \text { ； } \\
& T_{1}=\frac{C}{h} z \\
& P_{1}=\frac{g \beta C}{2 h}\left(z^{2}-h^{2}\right) \\
& T_{2}=\frac{D}{h} z+\frac{\Omega z C}{6 h \chi}\left(z^{2}-h^{2}\right) \\
& P_{2}=\frac{g \beta D}{2 h}\left(z^{2}-h^{2}\right)+\frac{g \beta \Omega C}{24 h \chi}\left(z^{4}-2 h^{2} z^{2}+h^{4}\right) \\
& V=W \sin \alpha-\frac{D \sigma}{\eta} z+\frac{g \beta D z}{24 h v}\left(z^{3}-6 h^{2} z+8 h^{3}\right)-\frac{g \beta \Omega C}{720 h v \chi} z\left(z^{5}+5 h^{2} z^{3}-15 h^{4} z+16 h^{5}\right) \\
& U=W \cos \alpha-\frac{C \sigma}{\eta} z+\frac{g \beta C}{24 h v} z\left(z^{3}-6 h^{2} z+8 h^{3}\right)-\frac{g \beta \Omega D}{720 h v^{2}} z\left(z^{5}+15 h^{2} z^{3}-40 h^{3} z^{2}+66 h^{5}\right)- \\
& -\frac{W \sin \alpha \Omega}{2 v}(2 h-z) z+\frac{D \sigma \Omega}{6 \eta v}\left(3 h^{2}-z^{2}\right) z+\frac{g \beta \Omega^{2} C z}{120960 h v^{2} \chi}\left[3 z^{7}-28 h^{2} z^{5}+\right. \\
& \left.+210 h^{4} z^{3}-448 h^{5} z^{2}+648 h^{7}\right] \\
& T_{0}=\frac{z \vartheta}{h}-\frac{g \beta\left(C^{2}+D^{2}\right)}{5040 h^{2} v \chi}(h-z) z\left[5 z^{5}+5 h z^{4}-58 h^{2} z^{3}+82 h^{3} z^{2}+82 h^{4} z+82 h^{5}\right]- \\
& -\frac{W z}{6 h \chi}\left(D h^{2} \sin \alpha-(C \cos \alpha+D \sin \alpha) z^{2}\right)+\frac{\sigma\left(C^{2}+D^{2}\right)(h-z) z}{12 h \eta \chi}\left(z^{2}+h z+h^{2}\right)-
\end{aligned}
$$

Burmasheva N. V., Prosviryakov E. Yu. Temperature field investigation in layered flows of a vertically swirling viscous incompressible fluid under two thermocapillar forces at a free boundary // Diagnostics, Resource and Mechanics of materials and structures. - 2019. - Iss. 1. - P. 6-42. - DOI: 10.17804/2410-9908.2019.1.006-042. 


$$
\begin{gathered}
-\frac{g \beta \Omega C D(h-z) z}{120960 h^{2} v \chi^{2}}\left[14 z^{7}+14 h z^{6}-146 h^{2} z^{5}+78 h^{3} z^{4}+456 h^{4} z^{3}-328 h^{5} z^{2}-328 h^{6} z^{3}-328 h^{7}\right]- \\
-\frac{W \sin \alpha \Omega C(h-z) z}{360 h \chi^{2}}\left(3 z^{3}+3 h z^{2}-7 h^{2} z-7 h^{3}\right)+ \\
+\frac{C D \sigma \Omega(h-z) z}{360 h \eta \chi^{2}}\left(2 z^{4}+2 h z^{3}-3 h^{2} z^{2}-3 h^{3} z-3 h^{4}\right)-
\end{gathered}
$$$$
-\frac{g \beta \Omega C D(h-z) z}{362880 h^{2} v^{2} \chi}\left[7 z^{7}+7 h z^{6}-173 h^{2} z^{5}+499 h^{3} z^{4}+499 h^{4} z^{3}-2273 h^{5} z^{2}-2273 h^{6} z-2273 h^{7}\right]-
$$$$
-\frac{W \sin \alpha \Omega C(h-z) z}{120 h v \chi}\left(3 z^{3}-7 h z^{2}-7 h^{2} z-7 h^{3}\right)-
$$$$
+\frac{C D \sigma \Omega(h-z) z}{360 h \eta v \chi}\left(2 z^{4}+2 h z^{3}-13 h^{2} z^{2}-13 h^{3} z-13 h^{4}\right)-
$$$$
-\frac{g \beta \Omega^{2} C^{2}(h-z) z}{33916800 h^{2} v \chi^{3}}\left[84 z^{9}+84 h z^{8}-686 h^{2} z^{7}-686 h^{3} z^{6}+3714 h^{4} z^{5}-1214 h^{5} z^{4}-\right.
$$$$
\left.-8144 h^{6} z^{3}+4176 h^{7} z^{2}+4176 h^{8} z+4176 h^{9}\right]-
$$$$
-\frac{g \beta \Omega^{2} C^{2}(h-z) z}{119750400 h^{2} v^{2} \chi^{2}}\left[27 z^{9}+27 h z^{8}-358 h^{2} z^{7}-358 h^{3} z^{6}+4592 h^{4} z^{5}-10192 h^{5} z^{4}-\right.
$$$$
\left.-10192 h^{6} z^{3}+43268 h^{7} z^{2}+43268 h^{8} z+43268 h^{9}\right]
$$$$
P_{0}=\frac{g^{2} \beta^{2}\left(C^{2}+D^{2}\right)(h-z)^{2}}{40320 h^{2} v \chi}\left[5 z^{6}+10 h z^{5}-69 h^{2} z^{4}+76 h^{3} z^{3}+221 h^{4} z^{2}+336 h^{5} z+183 h^{6}\right]-
$$$$
-\frac{g \beta \vartheta(h-z)(h+z)}{2 h}+\frac{W g \beta(C \cos \alpha+D \sin \alpha)(h-z)^{2}}{24 h \chi}\left[z^{2}+2 h z+h^{2}\right]-
$$$$
-\frac{g \beta \sigma\left(C^{2}+D^{2}\right)(h-z)^{2}}{120 h \eta \chi}\left[2 z^{3}+4 h z^{2}+6 h^{2} z+3 h^{3}\right]+
$$$$
+\frac{g^{2} \beta^{2} \Omega C D(h-z)^{2}}{1209600 h^{2} v \chi^{2}}\left[14 z^{8}+28 h z^{7}-158 h^{2} z^{6}-24 h^{3} z^{5}+740 h^{4} z^{4}-64 h^{5} z^{3}-868 h^{6} z^{2}-1671 h^{7} z-\right.
$$$$
\left.-836 h^{8}\right]+\frac{g \beta W \sin \alpha \Omega C(h-z)^{2}}{720 h \chi^{2}}\left[4 z^{4}+2 h z^{3}-2 h^{2} z^{2}-6 h^{3} z-3 h^{4}\right]-
$$

Burmasheva N. V., Prosviryakov E. Yu. Temperature field investigation in layered flows of a vertically swirling viscous incompressible fluid under two thermocapillar forces at a free boundary // Diagnostics, Resource and Mechanics of materials and structures. - 2019. - Iss. 1. - P. 6-42. - DOI: 10.17804/2410-9908.2019.1.006-042. 


$$
\begin{gathered}
-\frac{g \beta C D \sigma \Omega(h-z)^{2}}{5040 h \eta \chi^{2}}\left[4 z^{5}+8 h z^{4}-2 h^{2} z^{3}-12 h^{3} z^{2}-22 h^{4} z-11 h^{5}\right]+ \\
+\frac{g^{2} \beta^{2} \Omega C D(h-z)^{2}}{3628800 h^{2} v^{2} \chi}\left[7 z^{8}+14 h z^{7}-204 h^{2} z^{6}+538 h^{3} z^{5}+1280 h^{4} z^{4}-3522 h^{5} z^{3}-\right. \\
\left.-8324 h^{6} z^{2}-13126 h^{7} z-6563 h^{8}\right]+ \\
+\frac{g \beta W \sin \alpha \chi \Omega C(h-z)^{2}}{240 h v \chi}\left[z^{4}-2 h z^{3}-5 h^{2} z^{2}-8 h^{3} z-4 h^{4}\right]- \\
-\frac{g \beta C D \sigma \Omega(h-z)^{2}}{5040 h \eta v \chi}\left[4 z^{5}+8 h z^{4}-30 h^{2} z^{3}-68 h^{3} z^{2}-106 h^{4} z-53 h^{5}\right]+ \\
+\frac{g^{2} \beta^{2} \Omega^{2} C^{2}(h-z)^{2}}{79833600 h^{2} v \chi^{3}}\left[14 z^{10}+28 h z^{9}-112 h^{2} z^{8}-252 h^{3} z^{7}+708 h^{4} z^{6}+260 h^{5} z^{5}-\right. \\
\left.-2498 h^{6} z^{4}-328 h^{7} z^{3}+1842 h^{8} z^{2}+4012 h^{9} z+2006 h^{10}\right]+ \\
+\frac{g^{2} \beta^{2} \Omega^{2} C^{2}(h-z)^{2}}{479001600 h^{2} v^{2} \chi^{2}}\left[9 z^{10}+18 h z^{9}-127 h^{2} z^{8}-272 h^{3} z^{7}+2058 h^{4} z^{6}-4060 h^{5} z^{5}-\right. \\
\left.-1018 h^{6} z^{4}+26472 h^{7} z^{3}+63122 h^{8} z^{2}+99772 h^{9} z+49886 h^{10}\right] .
\end{gathered}
$$

В силу структуры выбранного класса решений (2) несимметричность выражений компонент $V_{x}, V_{y}$ поля скорости приводит к тому, что становится невозможным построить линейное преобразование, позволяющее снизить размерность исследуемой задачи, как это было проделано, например, в [34-38].

Поставим далее задачу исследования условий существования числа критических точек и возникновения вертикальной стратификации в температурном поле (аналогичное исследование для более простого класса скоростей было проведено авторами ранее, в частности в [36-38]).

\section{3. Исследование температурного поля при задании тепловых источников только на верхней границе}

Рассмотрим сначала предельный случай - случай задания однородного источника тепла ( $A=B=C=D=0$ ). Полагая все продольные градиенты температуры нулевыми, получаем, что компоненты температурного поля в изотермическом случае принимают вид:

$$
T_{1}=T_{2}=0, T_{0}=\vartheta Z
$$

Очевидно, что данное решение не может описывать стратификацию поля температуры.

Когда источник тепла не является однородным, поле температуры определяется вкладом нескольких потоков, индуцированных различными причинами, среди которых влияние термокапиллярного эффекта, неравномерный нагрев границ слоя жидкости, движение ниж- 
ней границы и др. Причем три из этих потоков индуцированы термокапиллярными силами в сочетании с другими факторами.

Введем всюду далее безразмерную координату $Z=z / h \in[0,1]$ и перепишем компоненты температурного поля с учетом введенной замены:

$$
\begin{aligned}
& T_{1}=C Z, T_{2}=Z\left[D+\frac{\Omega h^{2} C}{6 \chi}\left(Z^{2}-1\right)\right] \\
& T_{0}=\left(\vartheta-\frac{W h^{2} D \sin \alpha}{6 \chi}\right) Z-\frac{g \beta h^{5}\left(C^{2}+D^{2}\right)}{5040 v \chi}(1-Z) Z\left[5 Z^{5}+5 Z^{4}-58 Z^{3}+82 Z^{2}+82 Z+82\right]+ \\
& +\frac{W h^{2}(C \cos \alpha+D \sin \alpha)}{6 \chi} Z^{3}+\frac{\sigma\left(C^{2}+D^{2}\right) h^{3}(1-Z) Z}{12 \eta \chi}\left(Z^{2}+Z+1\right)- \\
& -\frac{g \beta \Omega C D h^{7}(1-Z) Z}{120960 v \chi^{2}}\left[14 Z^{7}+14 Z^{6}-146 Z^{5}+78 Z^{4}+456 Z^{3}-328 Z^{2}-328 Z^{3}-328\right]- \\
& -\frac{W \sin \alpha \Omega h^{4} C(1-Z) Z}{360 \chi^{2}}\left(3 Z^{3}+3 Z^{2}-7 Z-7\right)+ \\
& +\frac{C D \sigma \Omega h^{5}(1-Z) Z}{360 \eta \chi^{2}}\left(2 Z^{4}+2 Z^{3}-3 Z^{2}-3 Z-3\right)- \\
& -\frac{g \beta \Omega C D h^{7}(1-Z) Z}{362880 v^{2} \chi}\left[7 Z^{7}+7 Z^{6}-173 Z^{5}+499 Z^{4}+499 Z^{3}-2273 Z^{2}-2273 Z-2273\right]- \\
& -\frac{W \sin \alpha \Omega C h^{4}(1-Z) Z}{120 v \chi}\left(3 Z^{3}-7 Z^{2}-7 Z-7\right)+ \\
& +\frac{C D \sigma \Omega h^{5}(1-Z) Z}{360 \eta v \chi}\left(2 Z^{4}+2 Z^{3}-13 Z^{2}-13 Z-13\right)- \\
& -\frac{g \beta \Omega^{2} C^{2} h^{9}(1-Z) Z}{33916800 v \chi^{3}}\left[84 Z^{9}+84 Z^{8}-686 Z^{7}-686 Z^{6}+3714 Z^{5}-1214 Z^{4}-\right. \\
& \left.-8144 Z^{3}+4176 Z^{2}+4176 Z+4176\right]- \\
& -\frac{g \beta \Omega^{2} C^{2} h^{9}(1-Z) Z}{119750400 v^{2} \chi^{2}}\left[27 Z^{9}+27 Z^{8}-358 Z^{7}-358 Z^{6}+4592 Z^{5}-10192 Z^{4}-\right. \\
& \left.-10192 Z^{3}+43268 Z^{2}+43268 Z+43268\right] .
\end{aligned}
$$

Ввиду несимметричности выражений компонент поля скорости компоненты $T_{1}(Z)$ и $T_{2}(Z)$ температурного поля оказываются линейно независимы. Действительно, пусть существует линейное преобразование такое, что градиенты $T_{1}$ и $T_{2}$ оказываются связаны:

$$
T_{1} \equiv \lambda T_{2}, \quad \lambda \neq 0
$$

Burmasheva N. V., Prosviryakov E. Yu. Temperature field investigation in layered flows of a vertically swirling viscous incompressible fluid under two thermocapillar forces at a free boundary // Diagnostics, Resource and Mechanics of materials and structures. - 2019. - Iss. 1. - P. 6-42. - DOI: 10.17804/2410-9908.2019.1.006-042. 
Подставив полученные выше выражения для градиентов, получим:

$$
\begin{gathered}
C Z \equiv \lambda Z\left[D+\frac{\Omega h^{2} C}{6 \chi}\left(Z^{2}-1\right)\right] \\
Z\left[\lambda D-\left(\lambda \frac{\Omega h^{2}}{6 \chi}+1\right) C+\lambda \frac{\Omega h^{2} C}{6 \chi} Z^{2}\right] \equiv 0
\end{gathered}
$$

В силу принципа неопределенных коэффициентов последнее тождество равносильно системе условий:

$$
\left\{\begin{array}{l}
\lambda D=\frac{\lambda \Omega h^{2}+6 \chi}{6 \chi} C, \\
\lambda \frac{\Omega h^{2} C}{6 \chi}=0 .
\end{array}\right.
$$

Данная система совместна только при $C=D=0$. В рассматриваемом частном случае $A=B=0$ выполнение условия $C=D=0$ приводит к предельному случаю однородного источника тепла, разобранному выше. Таким образом, ввести линейную замену, позволяющую снизить размерность задачи без вырождения в нуль градиентов температуры, не представляется возможным.

Исследуем теперь температурное поле на экстремум. Необходимое условие существования стационарной точки [78] поля температуры гласит, что точка $\left(x_{0}, y_{0}, Z_{0}\right)$ является стационарной, если в ней выполняется система условий:

$$
\frac{\partial T}{\partial x}=0 ; \frac{\partial T}{\partial y}=0 ; \frac{\partial T}{\partial Z}=0
$$

С учетом структуры класса (3) эти условия равносильны следующей системе:

$$
T_{1}=0 ; T_{2}=0 ; \frac{\partial}{\partial Z}\left(T_{0}+T_{1} x+T_{2} y\right)=0
$$

Очевидно, $Z_{0}=0$ удовлетворяет первым двум условиям. Подстановка этого значения в третье условие позволит определить подходящие $x_{0}$ и $y_{0}$.

Для проверки выполнения достаточного условия существования экстремума необходимо рассмотреть значение в стационарной точке $\left(x_{0}, y_{0}, Z_{0}\right)$ квадратичную форму, отвечающую второму дифференциалу функции температуры:

$$
d F_{T}(d x, d y, d Z)=\left.\frac{\partial^{2} T}{\partial x^{2}}\right|_{\left(x_{0}, y_{0}, Z_{0}\right)} d x^{2}+\left.\frac{\partial^{2} T}{\partial y^{2}}\right|_{\left(x_{0}, y_{0}, Z_{0}\right)} d y^{2}+\left.\frac{\partial^{2} T}{\partial Z^{2}}\right|_{\left(x_{0}, y_{0}, Z_{0}\right)} d Z^{2}+
$$

Burmasheva N. V., Prosviryakov E. Yu. Temperature field investigation in layered flows of a vertically swirling viscous incompressible fluid under two thermocapillar forces at a free boundary // Diagnostics, Resource and Mechanics of materials and structures. - 2019. - Iss. 1. - P. 6-42. - DOI: 10.17804/2410-9908.2019.1.006-042. 


$$
\begin{gathered}
+\left.\frac{\partial^{2} T}{\partial x \partial y}\right|_{\left(x_{0}, y_{0}, Z_{0}\right)} d x d y+\left.\frac{\partial^{2} T}{\partial y \partial Z}\right|_{\left(x_{0}, y_{0}, Z_{0}\right)} d y d Z+\left.\frac{\partial^{2} T}{\partial x \partial Z}\right|_{\left(x_{0}, y_{0}, Z_{0}\right)} d x d Z= \\
=\left(\left.\frac{d^{2} T_{0}}{d Z^{2}}\right|_{Z_{0}}+\left.\frac{d^{2} T_{1}}{d Z^{2}}\right|_{Z_{0}} x_{0}+\left.\frac{d^{2} T_{2}}{d Z^{2}}\right|_{Z_{0}} y_{0}\right) d Z^{2}+\left.\frac{d T_{1}}{d Z}\right|_{Z_{0}} d x d Z+\left.\frac{d T_{2}}{d Z}\right|_{Z_{0}} d y d Z= \\
=\left.\frac{d^{2} T_{0}}{d Z^{2}}\right|_{Z_{0}} d Z^{2}+C d x d Z+\left(D-\frac{C \Omega h^{2}}{6 \chi}\right) d y d Z .
\end{gathered}
$$

Данная квадратичная форма ввиду сильной нелинейности фоновой температуры $T_{0}$ не является знакоопределенной, следовательно, экстремума не существует.

Исследуем теперь компоненты температурного поля на предмет наличия критических точек. Очевидно, что компонента $T_{1}(Z)$ не допускает расслоений, поскольку является строго монотонной (линейной) функцией. Несложно убедиться, что у компоненты $T_{2}(Z)$ может быть не более одного нуля на интервале $(0,1)$, так как в ее мультипликативную запись входит полином второй степени с ненулевым свободным членом, описывающий параболу с вершиной на границе исследуемого интервала $(0,1)$. Легко выписывается условие на параметры, при выполнении которого у градиента $T_{2}(Z)$ будет один нуль на исследуемом интервале. Для этого рассмотрим нули функции $T_{2}(Z)$ :

$$
T_{2}=0 \Leftrightarrow\left[\begin{array}{l}
Z=0, \\
D+\frac{\Omega h^{2} C}{6 \chi}\left(Z^{2}-1\right)=0 .
\end{array}\right.
$$

Точка $Z_{0}=0$ является нулем функции $T_{2}(Z)$, но не является критической. Выпишем корни получившегося квадратного уравнения:

$$
\frac{\Omega h^{2} C}{6 \chi} Z^{2}=\frac{\Omega h^{2} C}{6 \chi}-D
$$

Если $C \Omega=0$, градиент $T_{2}$ определяется выражением $T_{2}=D Z$ и, следовательно, при $D \neq 0$ не обращается в нуль внутри слоя жидкости. Полагая далее $C \Omega \neq 0$, приходим к уравнению

$$
Z^{2}=1-\frac{6 \chi D}{\Omega h^{2} C}
$$

Очевидно, что действительные корни существуют, если

$$
1-\frac{6 \chi D}{\Omega h^{2} C} \geq 0
$$


что равносильно следующему условию на управляющие параметры задачи:

$$
\frac{D}{\Omega C} \leq \frac{h^{2}}{6 \chi}
$$

Если последнее неравенство обратится в равенство (т. е. при $D=0$ ), будет единственный корень $-Z_{0}=0$. Если $\frac{D}{\Omega C} \leq 0$, то оба корня лежат вне исследуемого интервала $(0,1)$.

Изучим теперь поведение фоновой температуры $T_{0}(Z)$ и ее изменение по толщине слоя жидкости. Из приведенного выше точного решения видно, что фоновая температура описывается полиномом одиннадцатой степени с переменными коэффициентами. Известно [79], что число корней многочлена не превосходит его степени, в данном случае одиннадцати. Один корень выделяется явно - это $Z_{0}=0$, поскольку фоновая температура, очевидно, может быть представлена в мультипликативном виде:

$$
T_{0}=F(Z) Z,
$$

где $F(Z)$ есть линейная комбинация вида

$$
F=k_{0}+\sum_{i} k_{i} t_{i}(Z)
$$

Остальные десять корней многочлена $F(Z)$ могут лежать вне исследуемого интервала $(0,1)$ или же быть комплексными.

Очевидно, что любая критическая точка $Z_{j} \neq 0(j=\overline{1, m})$ фоновой температуры $T_{0}(Z)$ является также нулем функции $F(Z)$, так как справедливо равенство:

$$
0=T_{0}\left(Z_{j}\right)=F\left(Z_{j}\right) Z_{j}
$$

Что в свою очередь приводит к равенству

$$
k_{0}+\sum_{i} k_{i} t_{i}\left(Z_{j}\right)=0 \quad(j=\overline{1, m})
$$

Эти $m$ условий можно рассматривать как систему $m$ однородных уравнений относительно коэффициентов $k_{i}$. Причем среди строк матрицы получившейся линейной системы нет пропорциональных в силу строгой монотонности функций $t_{i}(Z)$ на интервале $(0,1)$. У такой системы всегда существует нетривиальное решение, если число условий $m$ меньше числа неизвестных, в роли которых выступают коэффициенты $k_{i}$.

В качестве иллюстрации приведенных выше рассуждений представлены профили фоновой температуры $T_{0}(Z)$ при различном числе критических точек у нее (рис. $1-10$ ). 


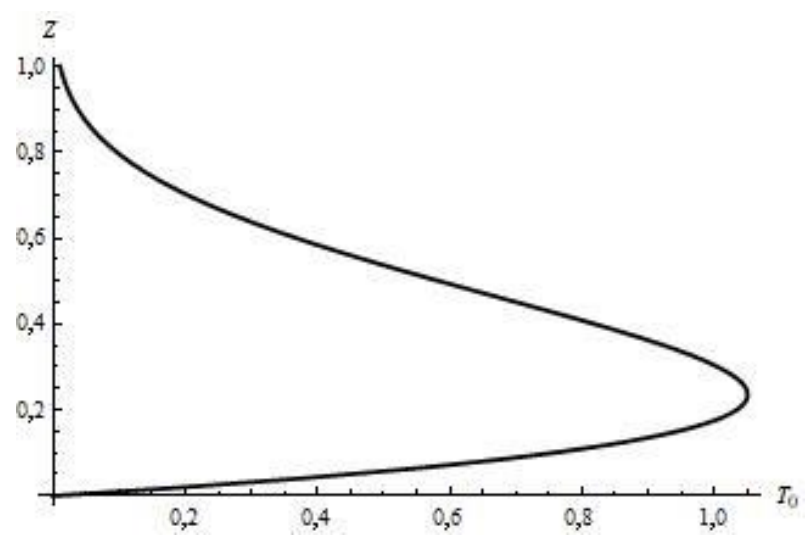

Рис. 1. Профиль температуры $T_{0}$ при отсутствии у нее критических точек

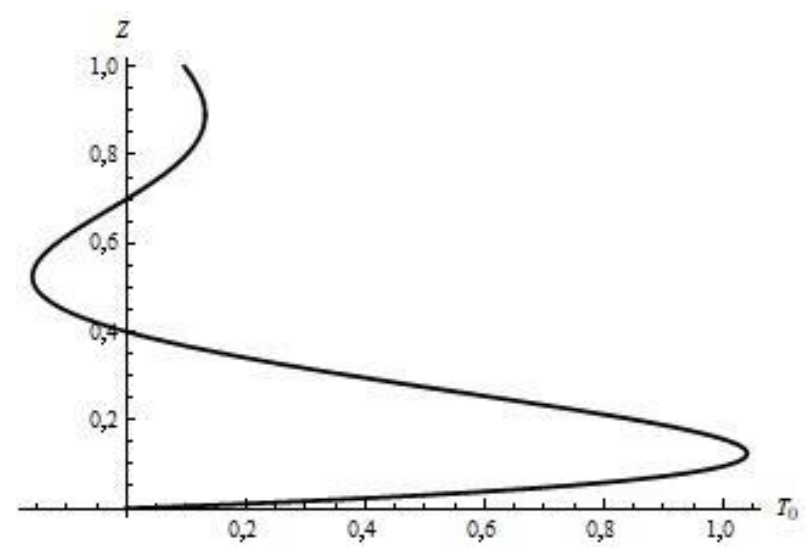

Рис. 3. Профиль температуры $T_{0}$ при наличии двух критических точек

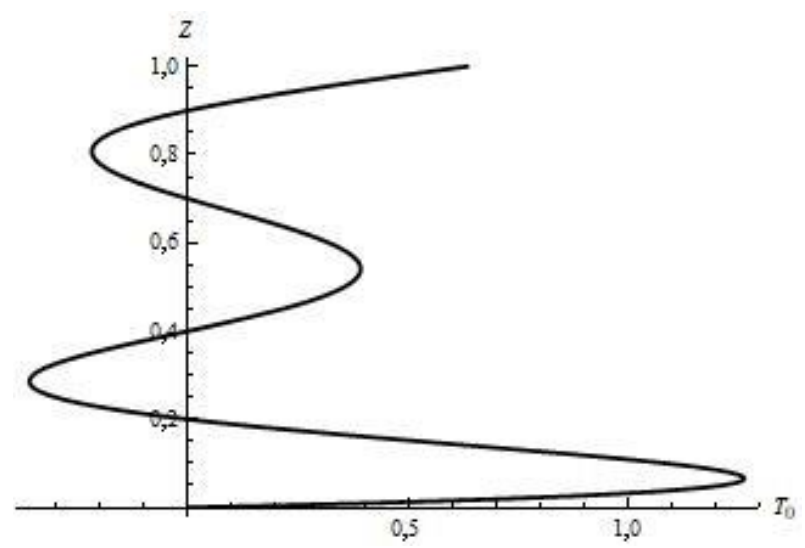

Рис. 5. Профиль температуры $T_{0}$ при наличии четырех критических точек

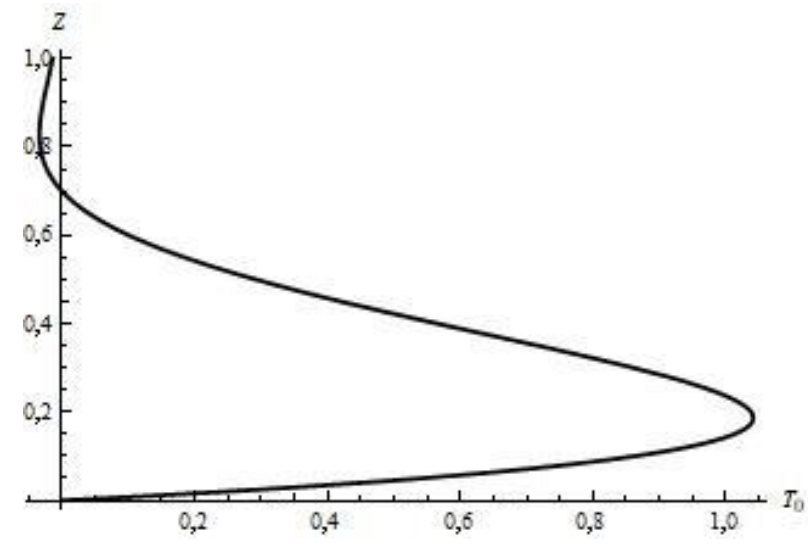

Рис. 2. Профиль температуры $T_{0}$ при наличии единственной критической точки

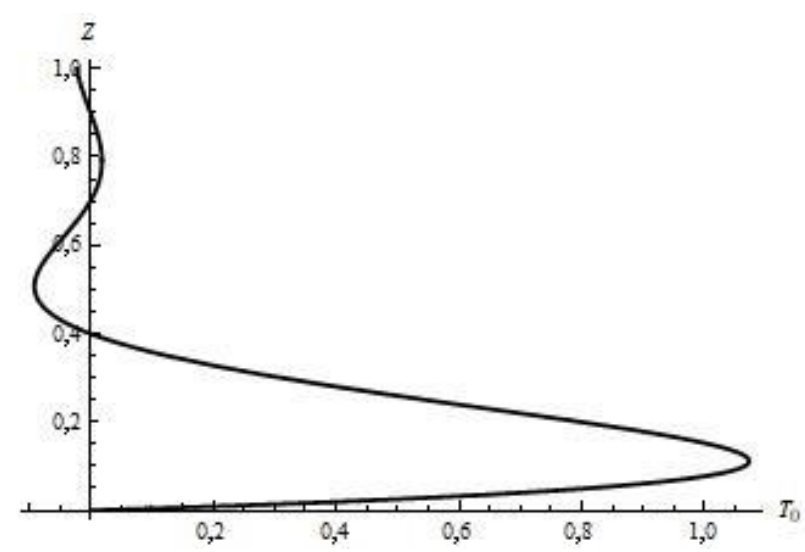

Рис. 4. Профиль температуры $T_{0}$ при наличии трех критических точек

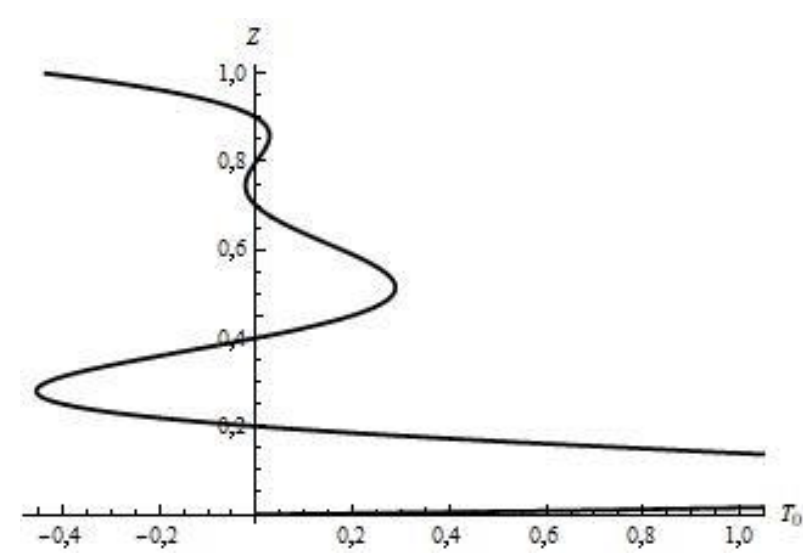

Рис. 6. Профиль температуры $T_{0}$ при наличии пяти критических точек 


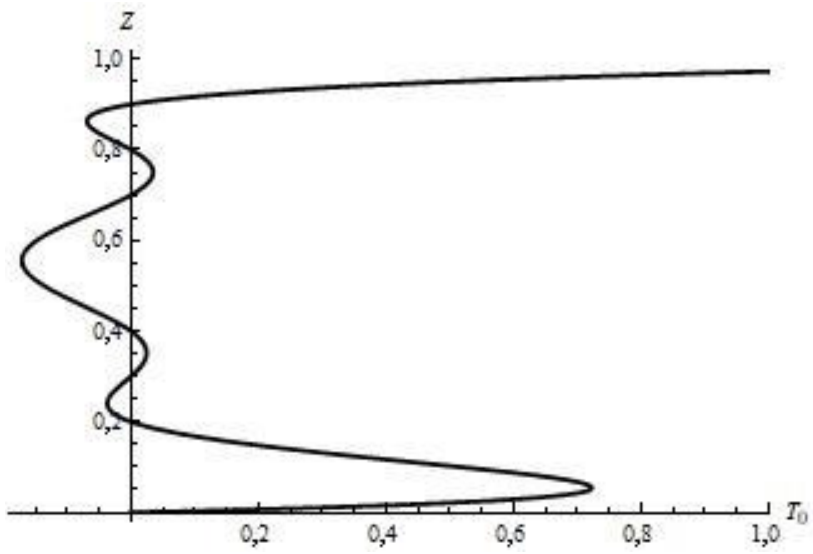

Рис. 7. Профиль температуры $T_{0}$ при наличии шести критических точек

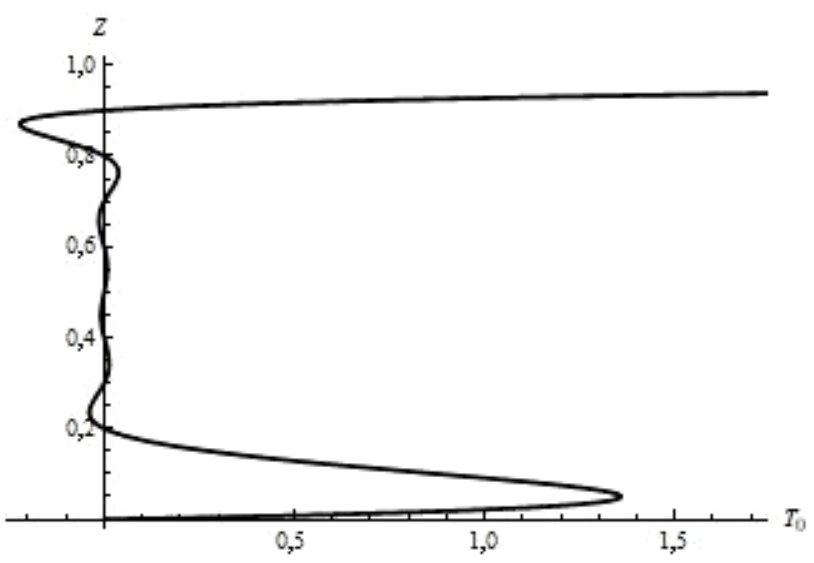

Рис. 9. Профиль температуры $T_{0}$ при наличии восьми критических точек

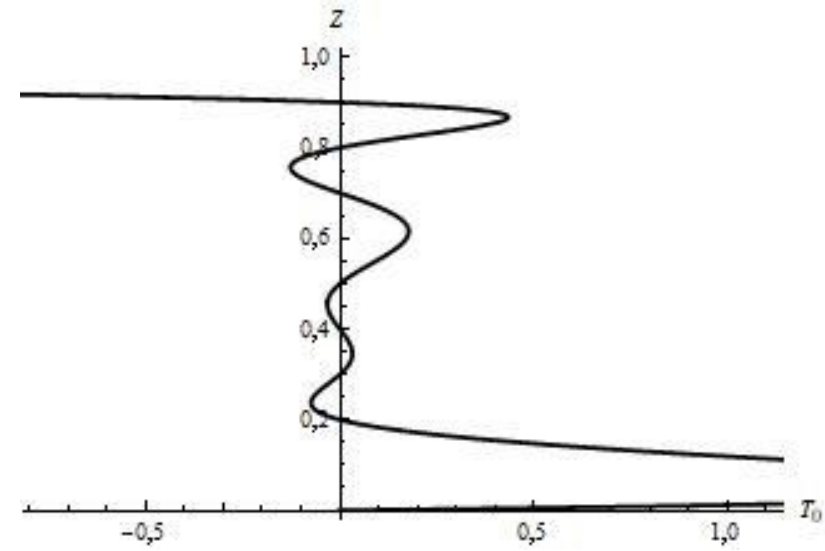

Рис. 8. Профиль температуры $T_{0}$ при наличии семи критических точек

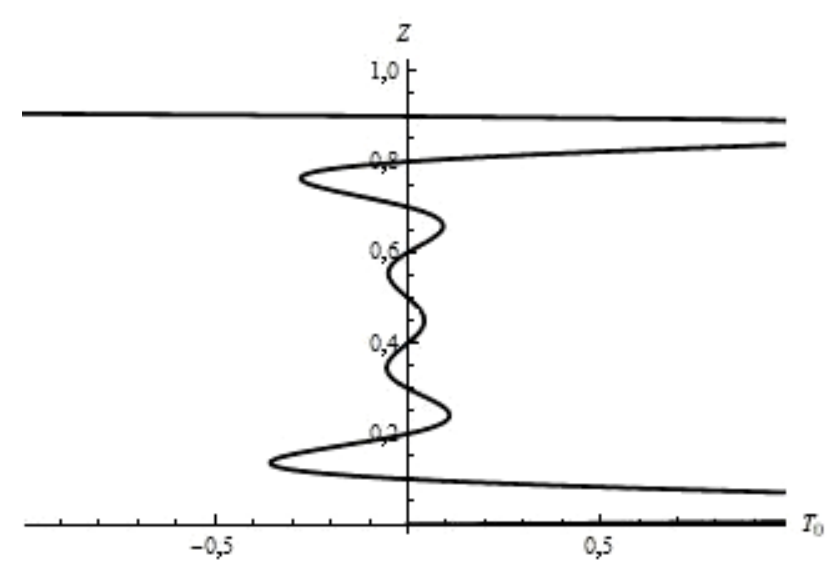

Рис. 10. Профиль температуры $T_{0}$ при наличии девяти критических точек

Рассмотрим далее поведение температурного поля $T$ с учетом рассмотренных выше особенностей его отдельных составляющих $T_{0}, T_{1}$ и $T_{2}$. Визуализация данного поля в общем случае не представляется возможной, так как температурному полю отвечает поверхность в четырехмерном пространстве. Чтобы снизить размерность, рассмотрим некоторые сечения, а точнее линии уровня температурного поля в двух характерных сечениях рассматриваемого слоя - $x=0$ (рис. 11 и 12) и $y=0$ (рис. 13). Как уже упоминалось выше, градиент $T_{1}(Z)$ не допускает расслоений, а у градиента $T_{2}(Z)$ может быть не более одного нуля на интервале $(0,1)$.

На рис. 11-12 четко видно, что изменение свойств температурного градиента $T_{2}$ (т. е. появление у него критической точки) качественным образом меняет распределение температуры по рассматриваемому сечению $x=0$. Прежде всего линии уровня меняют форму. Кроме того, пропадает ярко выраженная центральная зона, позволяющая разделить линии уровня на четыре группы в соответствии с их локализацией в сечении (рис. 11); линии уровня становятся качественно подобными, все сечение $x=0$ заполнено однотипными кривыми (рис. 12). 


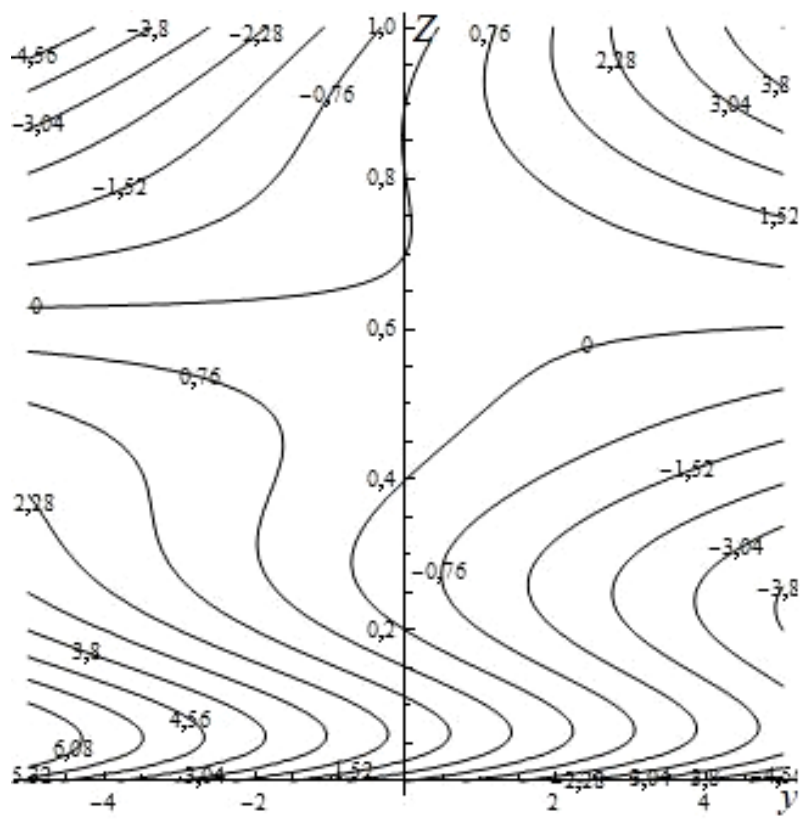

Рис. 11. Распределение линий уровня температурного поля в сечении $x=0$ (у фоновой температуры $T_{0}$ пять критических точек, у градиента $T_{2}$ их нет)

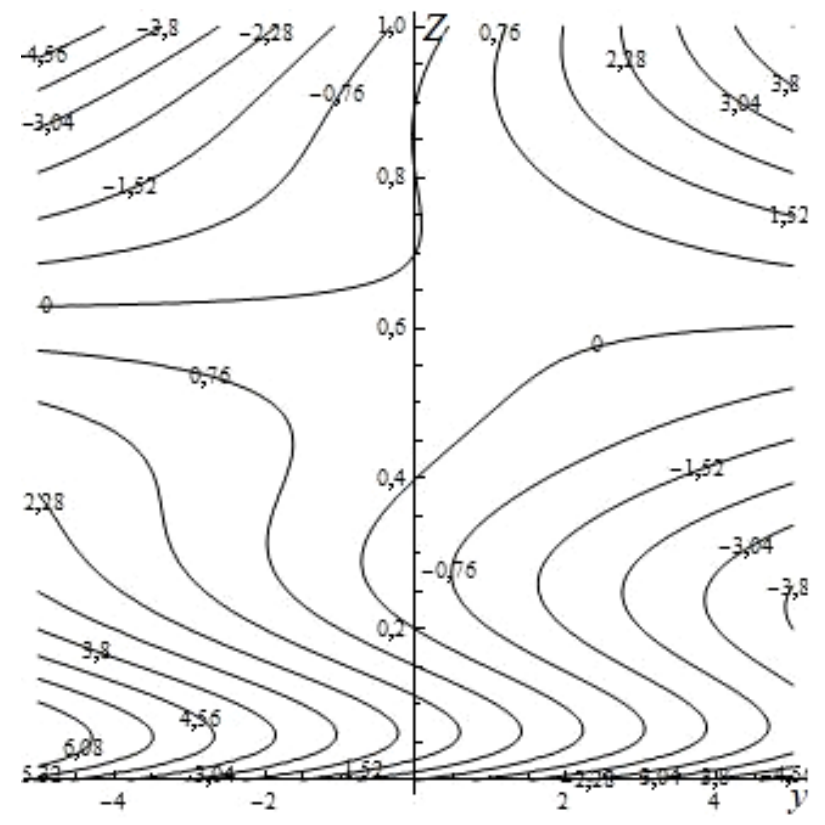

Рис. 12. Распределение линий уровня температурного поля в сечении $x=0$ (у фоновой температуры $T_{0}$ пять критических точек, у градиента $T_{2}$ - одна)

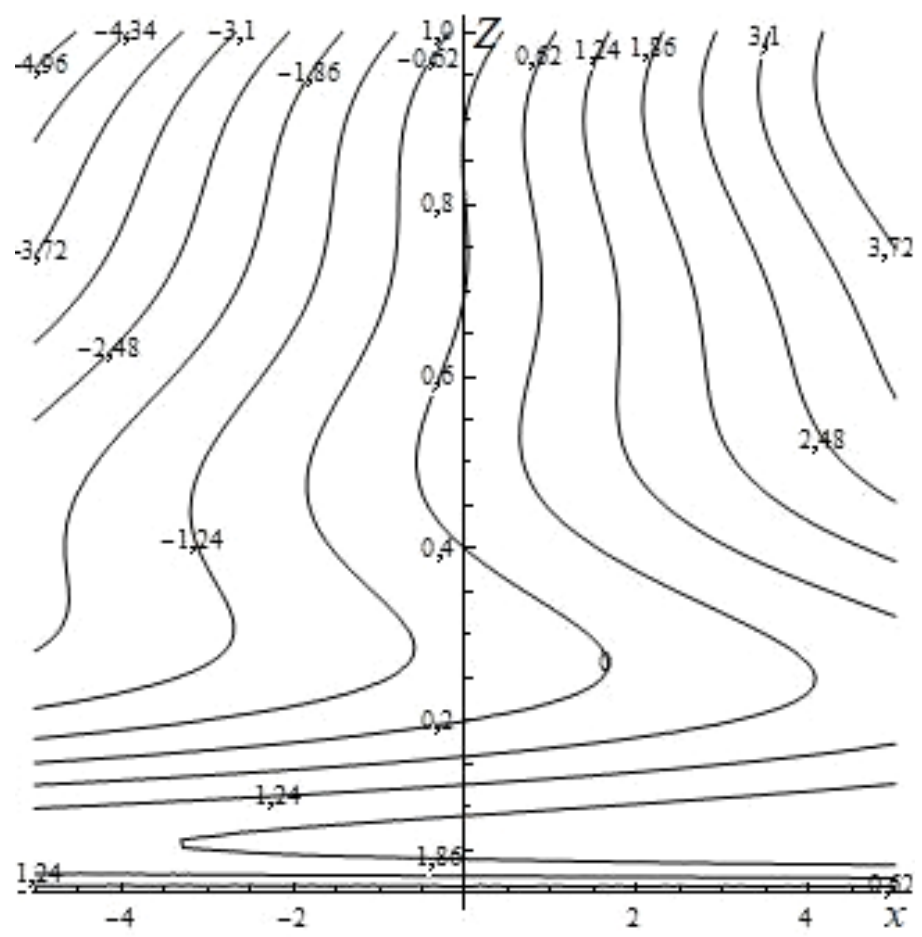

Рис. 13. Распределение линий уровня температурного поля в сечении $y=0$ (у фоновой температуры $T_{0}$ пять критических точек)

На рис. 14-17 представлено изменение распределения температурного поля при переходе от сечения $x=-0,7$ к сечению $x=0,7$ при отсутствии критических точек у градиента $T_{2}$; на рис. 18-21 - при наличии одной критической точки у градиента $T_{2}$. 


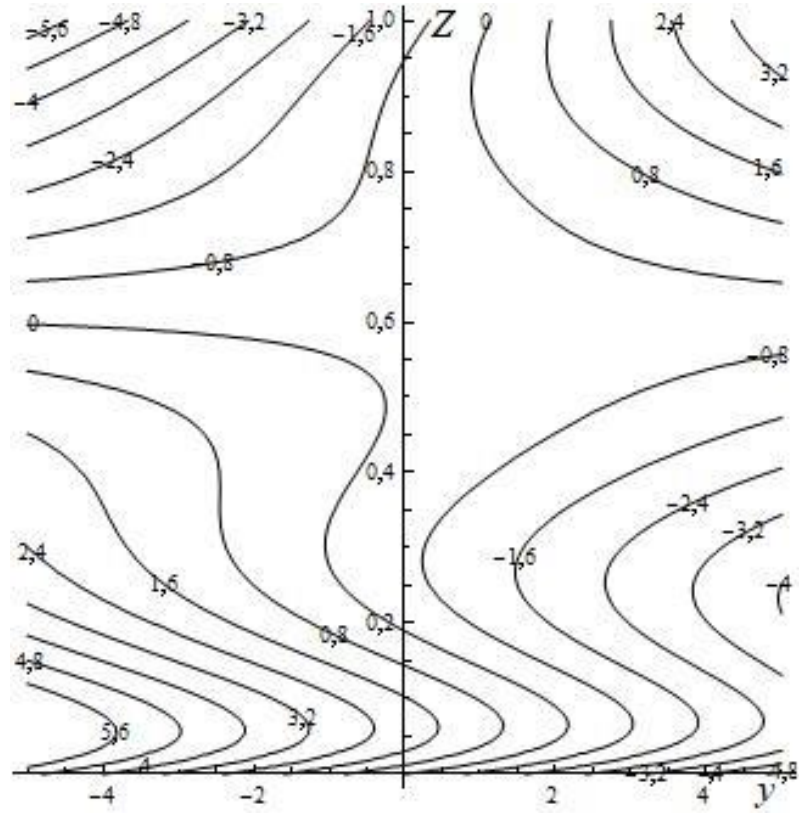

Рис. 14. Распределение линий уровня температурного поля в сечении $x=-0,7$ (у фоновой температуры $T_{0}$ пять критических точек, у градиента $T_{2}$ их нет)

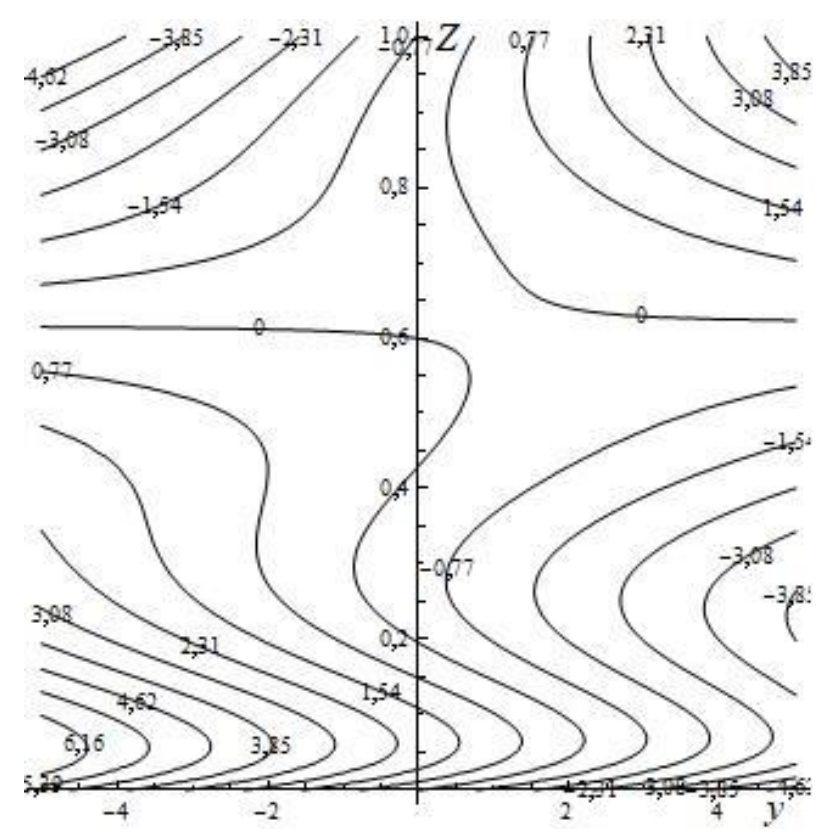

Рис. 15. Распределение линий уровня температурного поля в сечении $x=-0,3$ (у фоновой температуры $T_{0}$ пять критических точек, у градиента $T_{2}$ их нет)

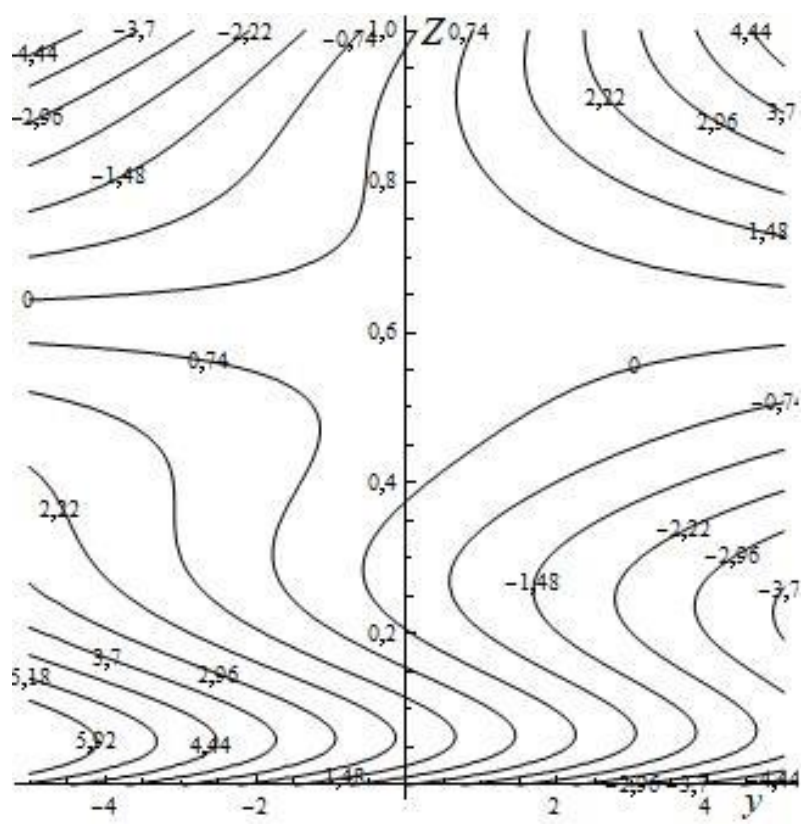

Рис. 16. Распределение линий уровня температурного поля в сечении $x=0,3$ (у фоновой температуры $T_{0}$ пять критических точек, у градиента $T_{2}$ их нет)

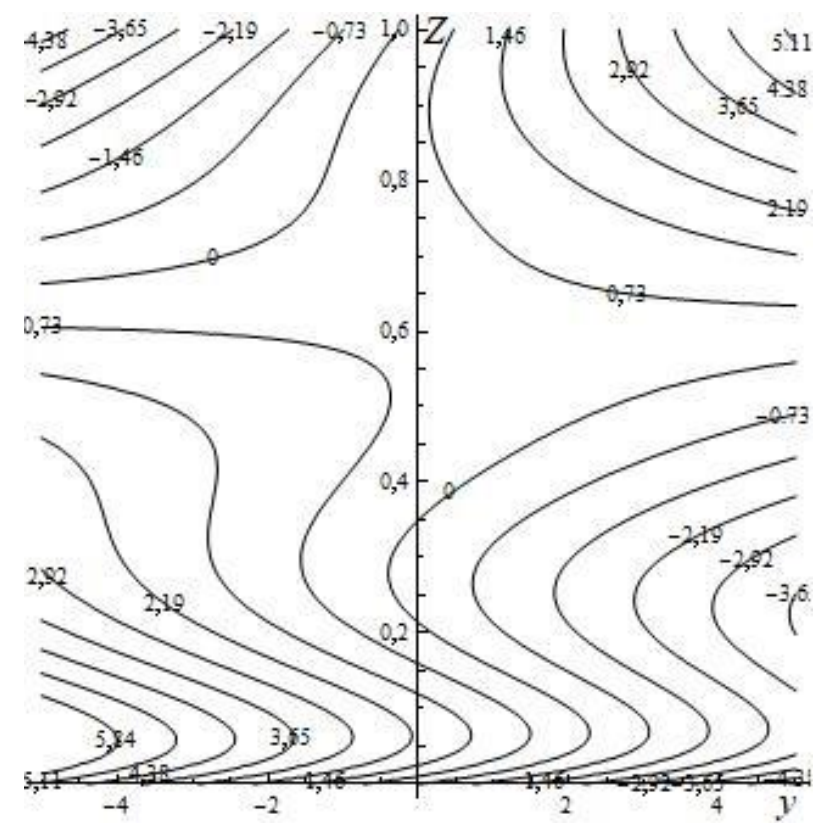

Рис. 17. Распределение линий уровня температурного поля в сечении $x=0,7$ (у фоновой температуры $T_{0}$ пять критических точек, у градиента $T_{2}$ их нет )

Из рис. 14-17 следует, что обнаруженная на рис. 11 центральная зона не пропадает при переходе от сечения к сечению вдоль оси $O x$. Она несколько деформируется, но тем не менее также позволяет разделять сечение на четыре подобласти, в каждой из которых линии уровня имеют подобную форму. 


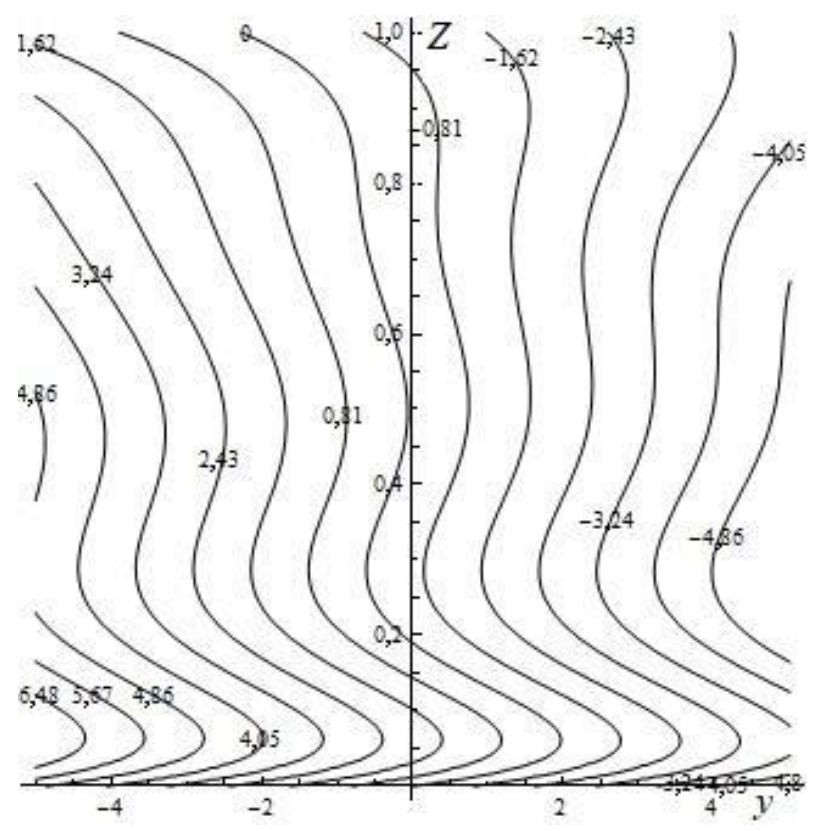

Рис. 18. Распределение линий уровня температурного поля в сечении $x=-0,7$ (у фоновой температуры $T_{0}$ пять критических точек, у градиента $T_{2}-$ одна критическая точка)

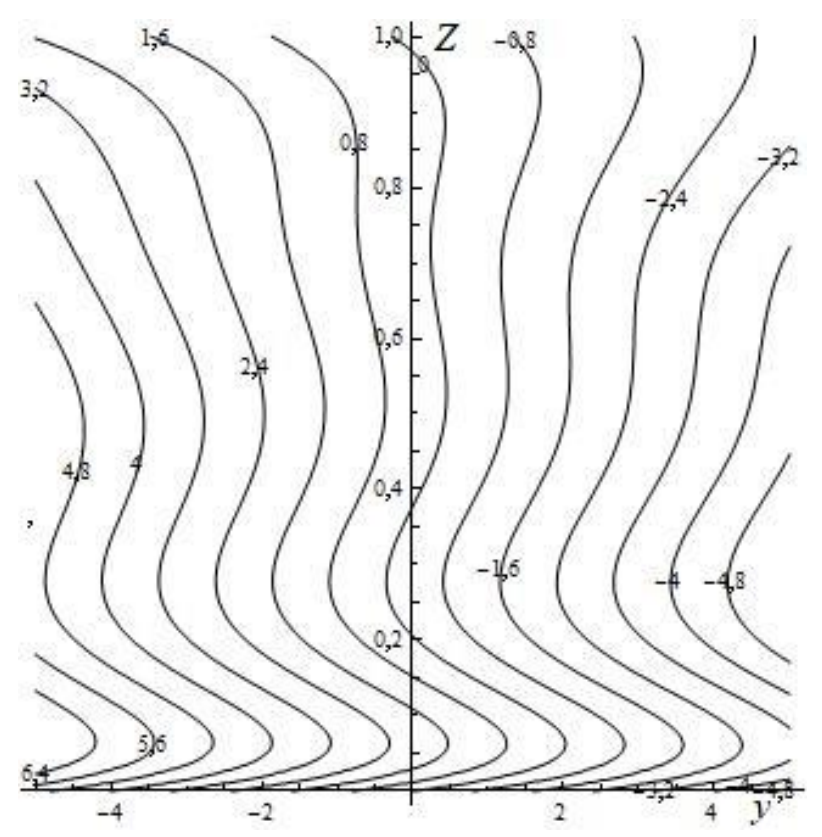

Рис. 20. Распределение линий уровня температурного поля в сечении $x=0,3$ (у фоновой температуры $T_{0}$ пять критических точек, у градиента $T_{2}$ - одна критическая точка)

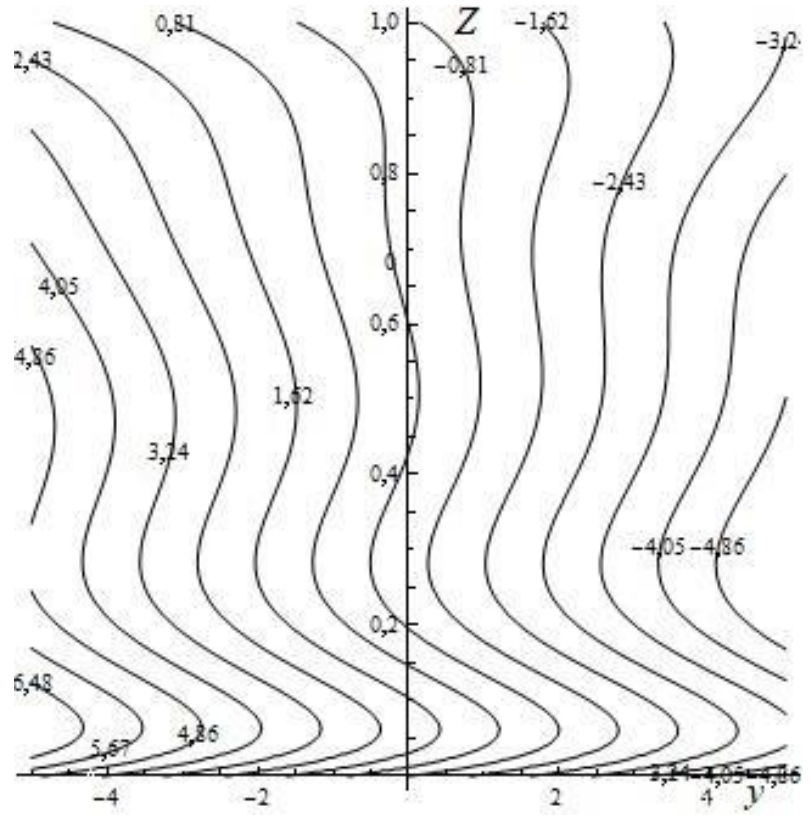

Рис. 19. Распределение линий уровня температурного поля в сечении $x=-0,3$ (у фоновой температуры $T_{0}$ пять критических точек, у градиента $T_{2}-$ одна критическая точка)

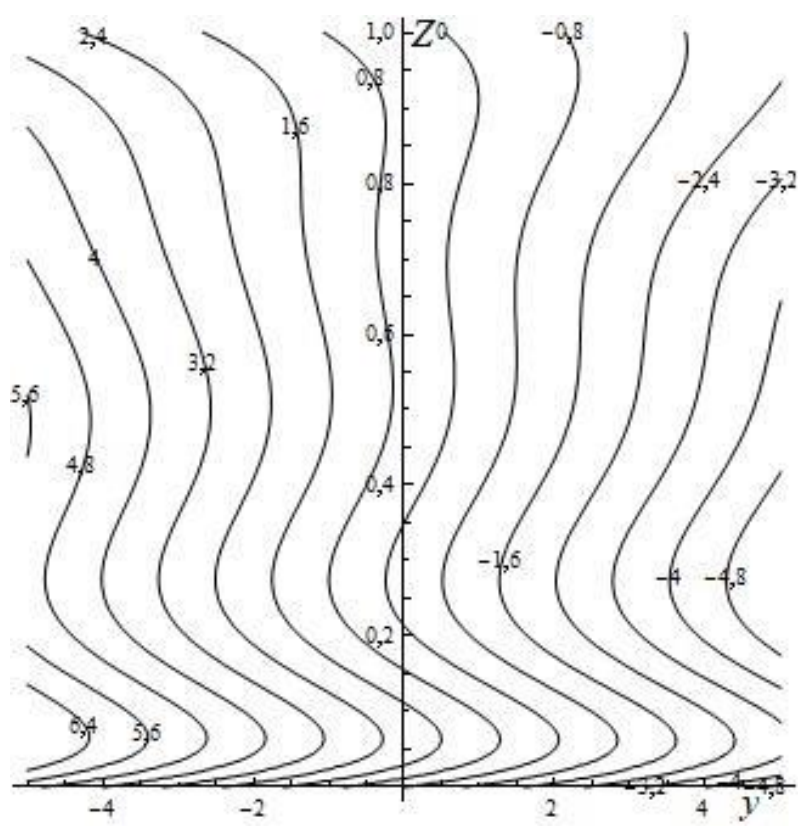

Рис. 21. Распределение линий уровня температурного поля в сечении $x=0,7$ (у фоновой температуры $T_{0}$ пять критических точек, у градиента $T_{2}$ - одна критическая точка)

Рис. 12, 18-21 демонстрируют качественное сохранение формы линий уровня и их движение по профилю при смене сечений.

Burmasheva N. V., Prosviryakov E. Yu. Temperature field investigation in layered flows of a vertically swirling viscous incompressible fluid under two thermocapillar forces at a free boundary // Diagnostics, Resource and Mechanics of materials and structures. - 2019. - Iss. 1. - P. 6-42. - DOI: 10.17804/2410-9908.2019.1.006-042. 


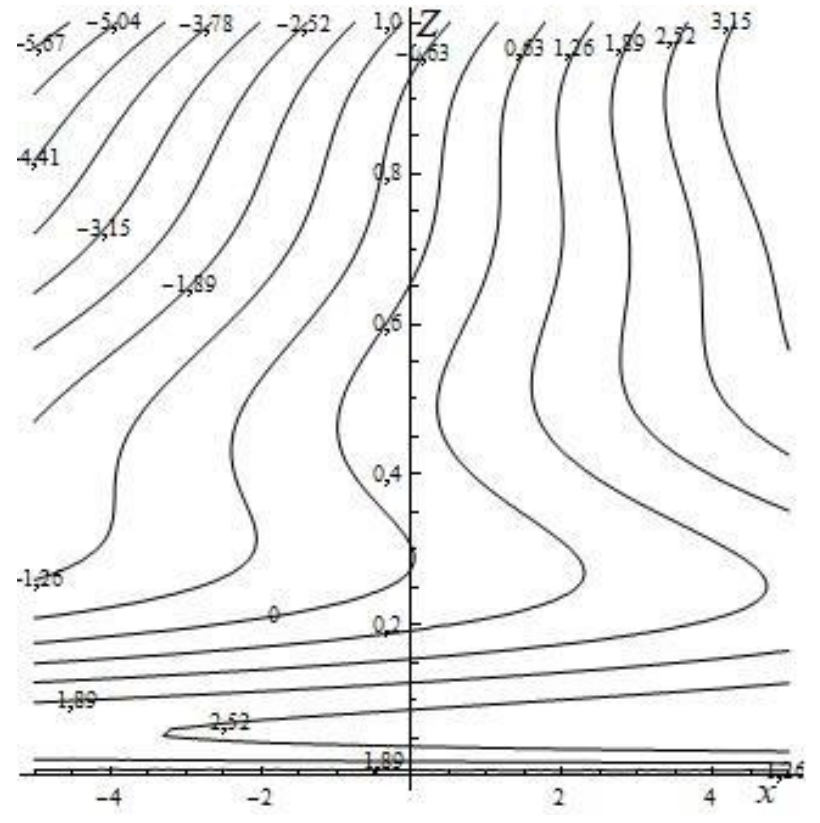

Рис. 22. Распределение линий уровня температурного поля в сечении $y=-0,7$ (у фоновой температуры $T_{0}$ пять критических точек, у градиента $T_{2}$ их нет)

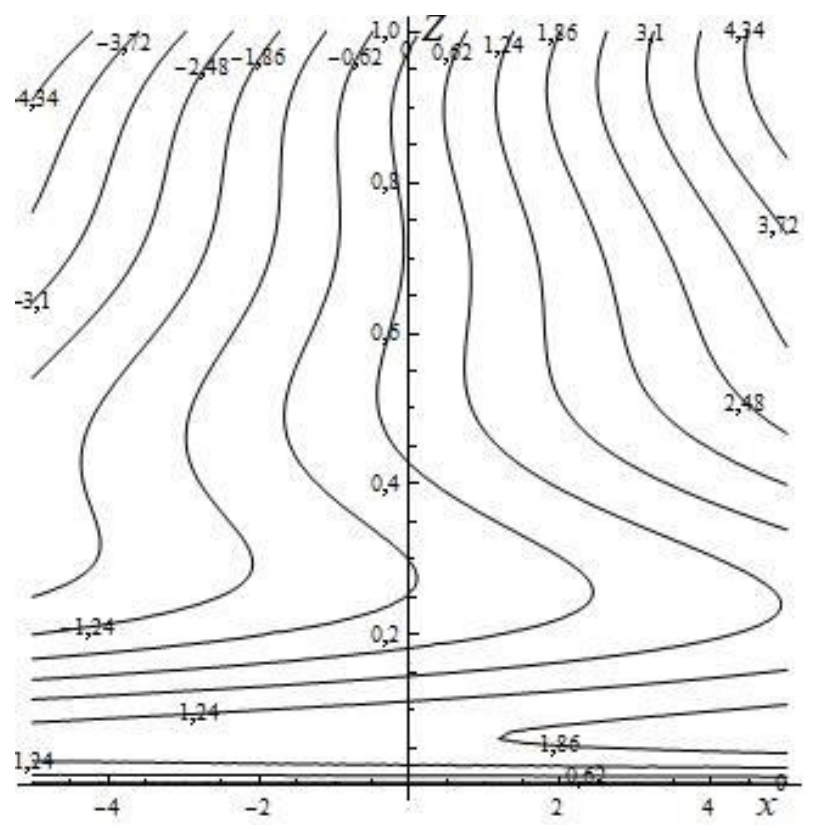

Рис. 24. Распределение линий уровня температурного поля в сечении $y=0,3$ (у фоновой температуры $T_{0}$ пять критических точек, у градиента $T_{2}$ их нет)

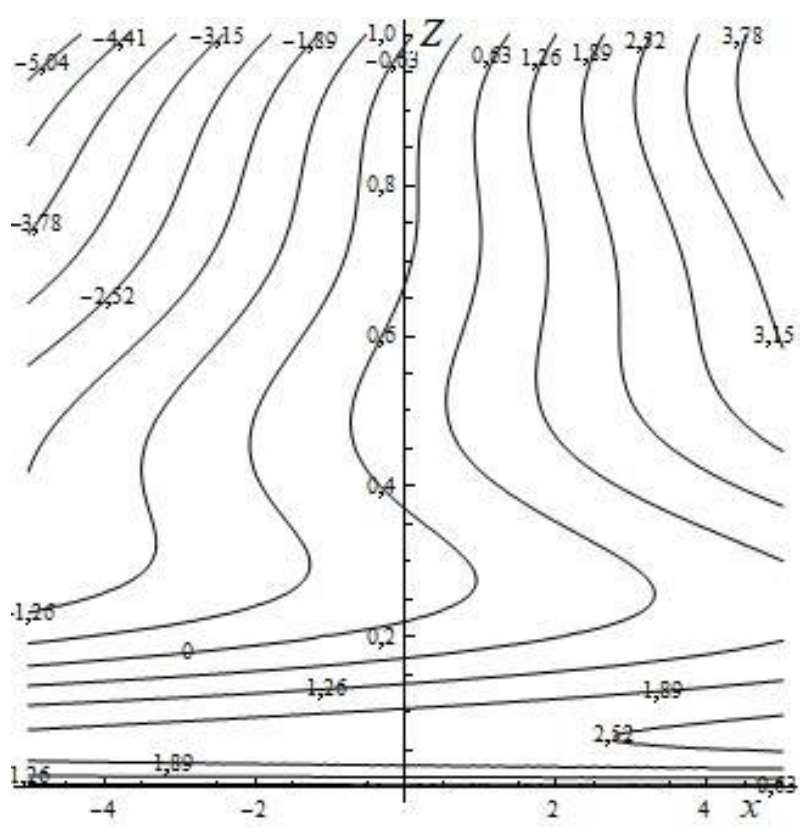

Рис. 23. Распределение линий уровня температурного поля в сечении $y=-0,3$ (у фоновой температуры $T_{0}$ пять критических точек, у градиента $T_{2}$ их нет)

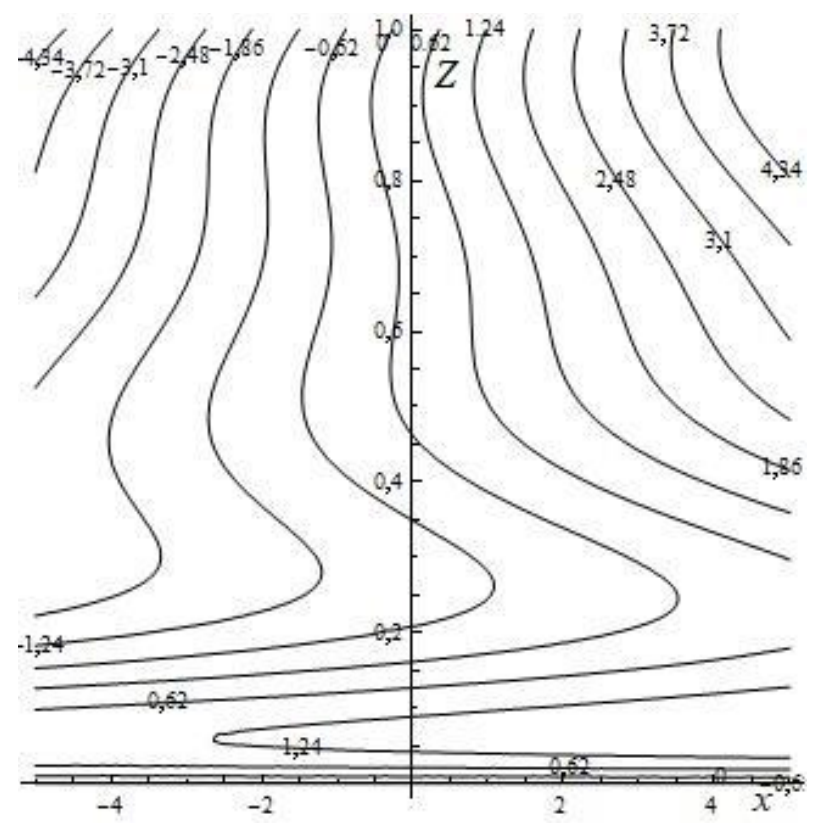

Рис. 25. Распределение линий уровня температурного поля в сечении $y=0,7$ (у фоновой температуры $T_{0}$ пять критических точек, у градиента $T_{2}$ их нет) 


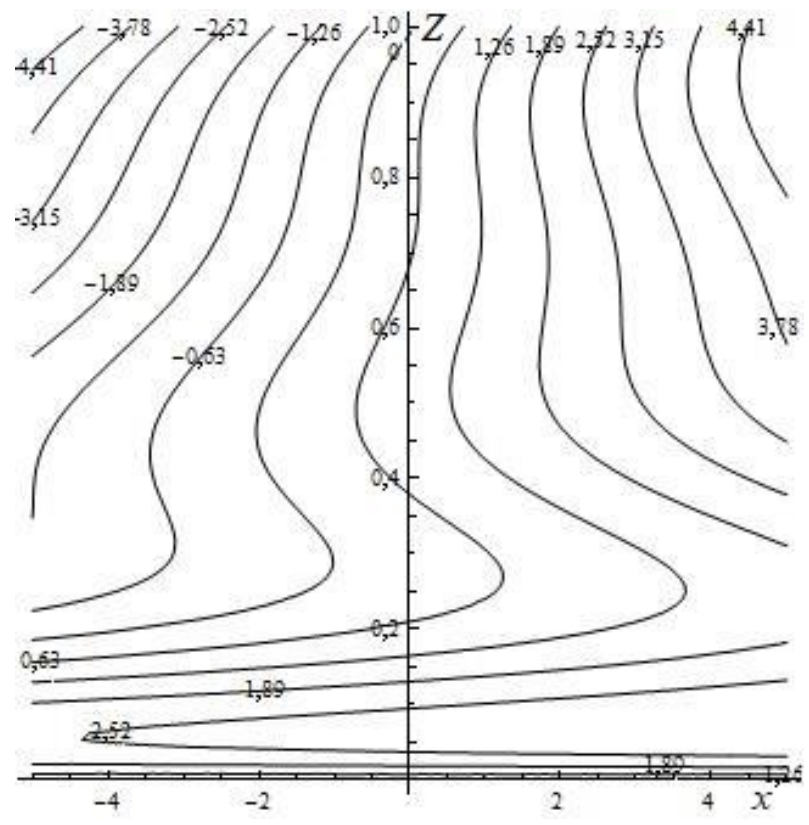

Рис. 26. Распределение линий уровня температурного поля в сечении $y=-0,7$ (у фоновой температуры $T_{0}$ пять критических точек, у градиента $T_{2}$ - одна критическая точка)

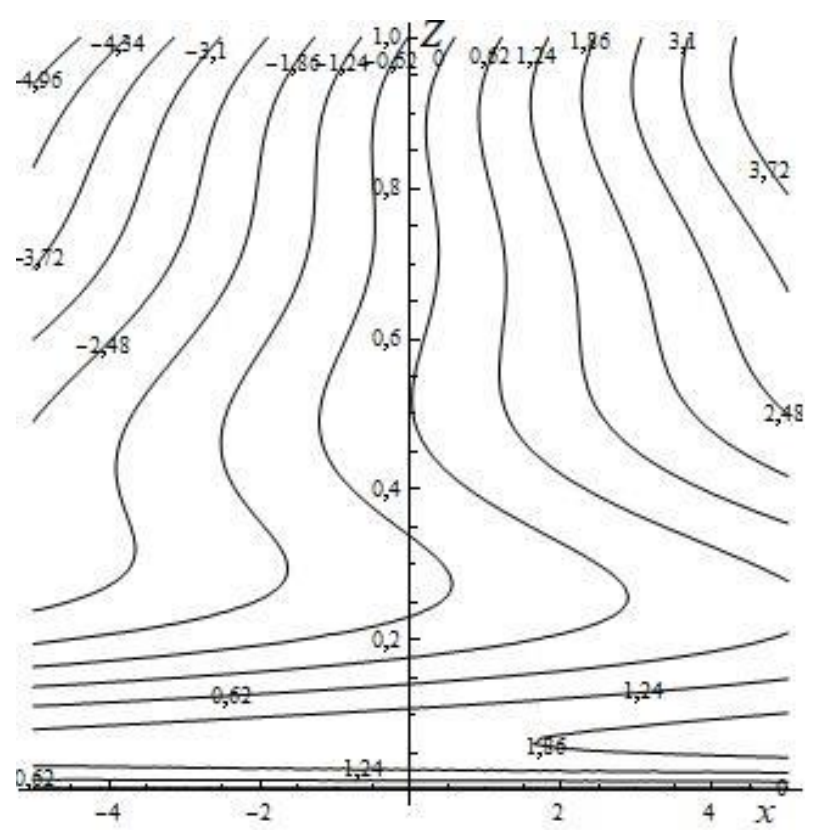

Рис. 28. Распределение линий уровня температурного поля в сечении $y=0,3$ (у фоновой температуры $T_{0}$ пять критических точек, у градиента $T_{2}-$ одна критическая точка)

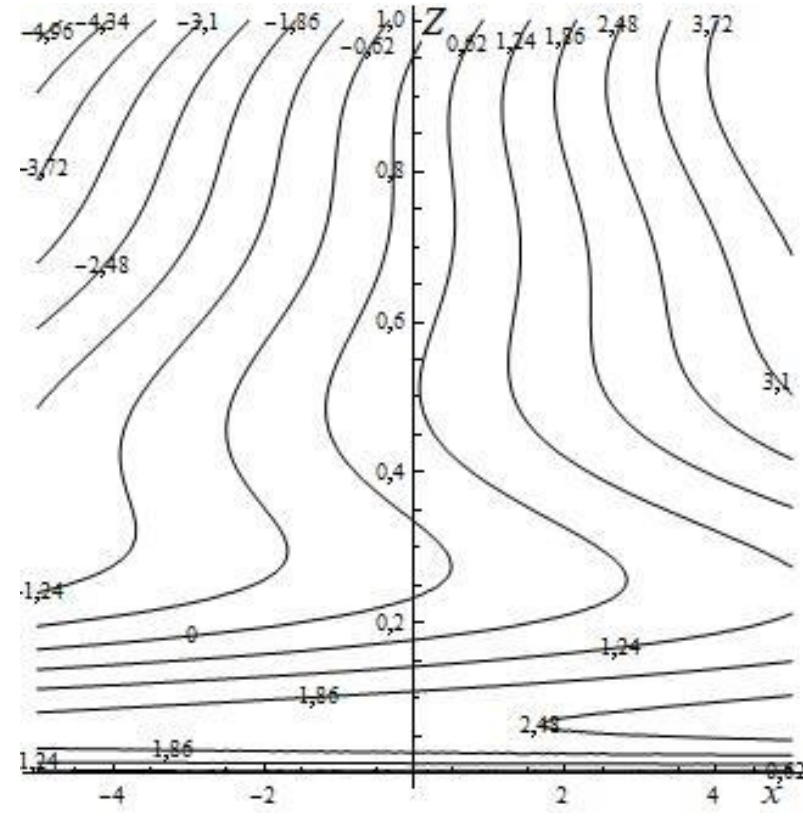

Рис. 27. Распределение линий уровня температурного поля в сечении $y=-0,3$ (у фоновой температуры $T_{0}$ пять критических точек, у градиента $T_{2}$ - одна критическая точка)

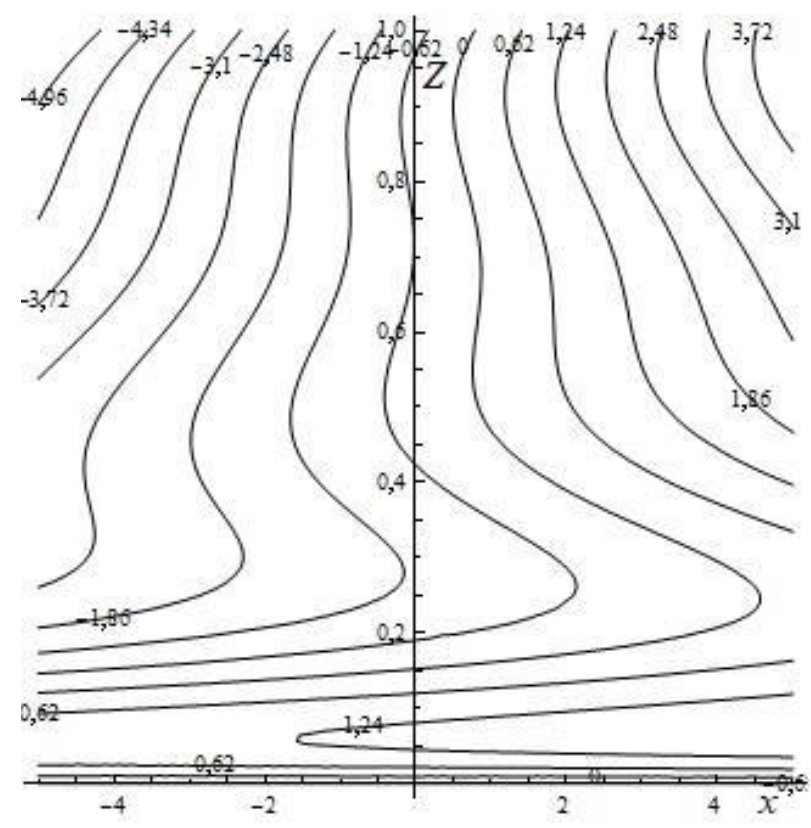

Рис. 29. Распределение линий уровня температурного поля в сечении $y=0,7$ (у фоновой температуры $T_{0}$ пять критических точек, у градиента $T_{2}-$ одна критическая точка) 
Аналогично изменение распределения температуры при переходе от сечения $y=-0,7$ к сечению $y=0,7$ при отсутствии критических точек у градиента $T_{2}$ приведено на рис. 22-25; на рис. 26-29 - при наличии одной критической точки у градиента $T_{2}$.

Сравнение рис. 22-25 и рис. 26-27 показывает, что линии уровня имеют качественно подобную форму и представленные профили отличаются только распределением значений поля температуры по этим линиям уровня. Из этого следует, что при данном сочетании управляющих параметров вклад градиента $T_{1}$ превалирует.

Непостоянство кривизны линий уровня в рассмотренных сечениях также подчеркивает факт существенно нелинейного характера распределения температуры в исследуемом слое. Отдельно отметим, что в случаях, когда термокапиллярный эффект вырождается, структура температурного поля становится несколько более простой, но также отражает нелинейность распределения температурного поля.

\section{4. Заключение}

В настоящей работе рассмотрено влияние термокапиллярного эффекта на одной из границ слоя вязкой несжимаемой жидкости на свойства слоистых крупномасштабных течений, индуцированных градиентами температуры. Для выбранных краевых условий было получено точное решение системы уравнений тепловой конвекции в приближении Буссинеска. Внимание в статье уделено температурному полю. Для полученного решения, описывающего изменение температуры, было показано, что соответствующая поверхность в четырехмерном фазовом пространстве не имеет точек экстремума. Кроме того, были исследованы на предмет возможных расслоений все компоненты температурного поля. Было показано, что один из градиентов температуры ни при каких условиях не обращается в нуль внутри исследуемого слоя жидкости, а для остальных компонент при некоторых условиях найдется толщина слоя жидкости, при которой в слое появляются точки, при переходе через которые эти компоненты меняют знак на противоположный. Показано, что такие точки могут быть не единственны.

\section{Литература}

1. Гершуни Г. 3., Жуховицкий Е. М. Конвективная неустойчивость несжимаемой жидкости. - М. : Наука, 1972. - 532 с.

2. Ekman V. W. On the Influence of the Earth's Rotation on Ocean-Currents // Ark. Mat. Astron. Fys. - 1905. - Vol. 2, no. 11. - P. 1-52.

3. Hiemenz K. Die Grenzschicht an einem in den gleichfцrmigen Flıssigkeitsstrom eingetauchten geraden Kreiszylinder // Dingler's Politech. J. - 1911. - Vol. 326. P. 321-324.

4. Ekman V. W. Uber Horizontazirkulation bei winder-reugten Meeresstromungen // Arkiv Mat., Astr., Phys. - 1923. - Vol. 17, no. 26. - P. 1-74.

5. Pedlosky J. Geophysical Fluid Dynamics. - New York, Heidelberg, Berlin : SpringerVerlag, 1987. - $710 \mathrm{p}$.

6. Haeusser T. M., Leibovich S. Pattern formation in the marginally unstable Ekman layer // J. Fluid Mech. - 2003. - Vol. 479. - P. 125-144. - DOI: 10.1017/S0022112002003415.

7. Schwarz K. G. Stability of thermocapillary advective flow in a slowly rotating liquid layer under microgravity conditions // Fluid Dynamics. - 2012. - Vol. 47, iss. 1. - P. 37-49. DOI: $10.1134 / \mathrm{S} 001546281201005 X$.

8. Aristov S. N., Frik P. G. Nonlinear effects of the Ekman layer on the dynamics of largescale eddies in shallow water // J. Appl. Mech. Tech. Phys. - 1991. - Vol. 32, no. 2. - P. 189-194. 
9. Gorshkov A. V., Prosviryakov E. Y. Ekman Convective Layer Flow of a Viscous Incompressible Fluid // Izvestiya. Atmospheric and Oceanic Physics. - 2018. - Vol. 54, no. 2. - P. 189-195. DOI: $10.1134 / \mathrm{S} 0001433818020081$.

10. Крюков Н. Д., Шматков В. А. Обзор исследований поверхностных течений, генерируемых ветром // Евразийский союз ученых. - 2015. - № 6-2 (15). - С. 109-113.

11. Шварц К. Г. Об одном точном решении уравнений Навье-Стокса, описывающем неизотермическое крупномасштабное течение во вращающемся слое жидкости со свободной верхней границей // Вестник пермского университета. Математика. Механика. Информатика. - 2016. Вып. 2 (33). - С. 118-124.

12. Shvarts K. G. Exact solution of the Navier-Stokes equation describing nonisothermal largescale flows in a rotating layer of liquid with free upper surface // J. Math. Sci. - 2018. Vol. 230, no. 5. - P. 813-817. - DOI: 10.1007/s10958-018-3796-y.

13. Brown R. A. Analytical Methods in Planetary Boundary-Layer Modelling. - Hilger, 1974.

14. Welander P. The thermocline problem // Phil. Trans. R. Soc. Lond. A. - 1971. - Vol. 270. P. 415-421. - DOI: 10.1098/rsta.1971.0081.

15. Chefranov S. G. Cyclone-anticyclone vortex asymmetry mechanism and linear Ekman friction // Journal of Experimental and Theoretical Physics. - 2016. - Vol. 122, no. 4. - P. 759-768. DOI: $10.1134 /$ S1063776116040038.

16. Riabouchinsky D. Quelques considerations sur les mouvements plans rotationnels d'un liquide // C. R. Hebdomadaires Acad. Sci. - 1924. - Vol. 179. - P. 1133-1136.

17. Galaktionov V. A., Svirshchevskii S. R. Exact solutions and invariant subspaces of nonlinear partial differential equations in mechanics and physics. - Boca Raton : Chapman and Hall/CRC, 2007.

18. Пухначев В. В. Групповые свойства уравнений Навье-Стокса в плоском случае // ПМТФ. - 1960. - № 1. - С. 83-90.

19. Атлас осесимметричных кавитационных течений типа Рябушинского / АН СССР. Сиб. отд-ние. Ин-т гидродинамики. - Новосибирск, 1968. - Вып. 1.

20. Гузевский Л. Г. Осесимметричные задачи обтекания со свободными границами // Исследования по развитой кавитации : сб. - Новосибирск, СО АН СССР, Ин-т теплофизики, 1976.

21. Aristov S. N., Knyazev D. V. Viscous fluid flow between moving parallel plates // Fluid Dynamics. - 2012. - Vol. 47, iss. 4. - P. 476-482. - DOI: 10.1134/S0015462812040060.

22. Petrov A. G. Exact solution of the Navier-Stokes equations in a fluid layer between the moving parallel plates // Journal of Applied Mechanics and Technical Physics. - 2012. - Vol. 53, no. 5. P. 642-646. - DOI: 10.1134/S0021894412050021.

23. Власова С. С., Просвиряков Е. Ю. Плоская конвекция вязкой несжимаемой жидкости при заданной теплоотдаче на свободной границе // Вестн. Сам. гос. техн. ун-та. Сер. физ.мат. науки. - 2016. - Т. 20, № 3. - С. 567-577. - DOI: 10.14498/vsgtu1483.

24. Аристов С. Н., Просвиряков Е. Ю. Об одном классе аналитических решений стационарной осесимметричной конвекции Бенара-Марангони вязкой несжимаемой жидкости // Вестн. Сам. гос. техн. ун-та. Сер. Физ.-мат. Науки. - 2013. - 3 (32). - C. 110-118. - DOI: $10.14498 /$ vsgtu 1205 .

25. Aristov S. N., Privalova V., Prosviryakov E. Y. Stationary nonisothermal Couette flow. Quadratic heating of the upper boundary of the fluid layer // Rus. J. Nonlin. Dyn. - 2016. - Vol. 12, no. 2. - P. 167-178. - DOI: 10.20537/nd1602001. 
26. Fomin A. A, Fomina L. N. On the solution of fluid flow and heat transfer problem in a 2D channel with backward-facing step // Vestnik Samarskogo Gosudarstvennogo Tekhnicheskogo Universiteta. Seriya Fiziko-Matematicheskiye Nauki. - 2017. - Vol. 21, no. 2. - P. 362-375. DOI: $10.14498 /$ vsgtu 1545 .

27. Shtern V. Counterflows. Paradoxical Fluid Mechanics Phenomena. - Cambridge : Cambridge University Press, 2012. - 469 p. - DOI: 10.1017/CBO9781139226516.

28. Stuart J. T. The viscous flow near a stagnation point when the external flow has uniform vorticity // Journal of the Aerospace Sciences. - 1959. - Vol. 26, no. 2. - P. 124-125. DOI: $10.2514 / 8.7963$.

29. Dorrepaal J. M. An exact solution of the Navier-Stokes equation which describes nonorthogonal stagnation-point flow in two dimensions // Journal of Fluid Mechanics. - 1986. - Vol. 163, no. 1. - P. 141-147. - DOI: 10.1017/s0022112086002240.

30. Riesco-Chueca P., De la Mora J. F. Brownian motion far from equilibrium: a hypersonic approach // Journal of Fluid Mechanics - 1990. - Vol. 214. - P. 639-663. - DOI: 10.1017/ S0022112090000301.

31. Aristov S. N., Prosviryakov E. Yu. Nonuniform convective Couette flow // Fluid Dynamics. 2016. - Vol. 51, iss. 5. - P. 581-587. - DOI: 10.1134/S001546281605001X.

32. Горшков А. В., Просвиряков Е. Ю. Слоистая конвекция Бенара-Марангони при теплообмене по закону Ньютона-Рихмана // Компьютерные исследования и моделирование. 2016. - Т. 8, вып. 6. - С. 927-940.

33. Aristov S. N., Prosviryakov E. Y., Spevak L. F. Nonstationary laminar thermal and solutal Marangoni convection of a viscous fluid // Computational Continuum Mechanics. - 2015. - Vol. 8, no. 4. - P. 445-456. - DOI: 10.7242/1999-6691/2015.8.4.38.

34. Burmasheva N. V., Prosviryakov E. Yu. A large-scale layered stationary convection of an incompressible viscous fluid under the action of shear stresses at the upper boundary. Velocity field investigation // Journal of Samara State Technical University. Ser. Physical and Mathematical Sciences. - 2017. - Vol. 21, no. 1. - P. 180-196. - DOI: 10.14498/vsgtu1527.

35. Burmasheva N. V., Prosviryakov E. Yu. Exact solutions for layered large-scale convection induced by tangential stresses specified on the free boundary of a fluid layer // IOP Conference Series: Materials Science and Engineering. - 2017. - Vol. 208, conf. 1. - DOI: 10.1088/1757899X/208/1/012010.

36. Burmasheva N. V., Prosviryakov E. Yu. Exact solutions for natural convection of layered flows of a viscous incompressible fluid with specified tangential forces and the linear distribution of temperature on the layer boundaries // Diagnostics, Resource and Mechanics of materials and structures. - 2017. - Iss. 4. - P. 16-31. - DOI: 10.17804/2410-9908.2017.4.016-031. URL: http://dream-journal.org/DREAM_Issue_4_2017_Burmasheva_N.V._et_al._016_031.pdf 37. Burmasheva N. V., Prosviryakov E. Yu. Exact solution for the layered convection of a viscous incompressible fluid at specified temperature gradients and tangential forces on the free boundary // AIP Conference Proceedings. - 2017. - Vol. 1915, iss. 1. - DOI: 10.1063/1.5017353.

38. Бурмашева Н. В., Просвиряков Е. Ю. Крупномасштабная слоистая стационарная конвекция вязкой несжимаемой жидкости под действием касательных напряжений на верхней границе. Исследование полей температуры и давления // Вестн. Самарск. гос. техн. унив. Cер. физ.-мат. науки. - 2017. - Т. 21, №. 4. - C. 736-751. - DOI: 10.14498/vsgtu1568.

39. Aristov S. N., Knyazev D. V., Polyanin A. D. Exact solutions of the Navier-Stokes equations with the linear dependence of velocity components on two space variables // Theoretical 
Foundations of Chemical Engineering. - 2009. - Vol. 43, no. 5. - P. 642-662. DOI: $10.1134 / \mathrm{S} 0040579509050066$.

40. Ostroumov G. A. Free convection under the condition of the internal problem. - Washington, NACA Technical Memorandum 1407, National Advisory Committee for Aeronautics, 1958.

41. Birikh R. V. Thermocapillary convection in a horizontal layer of liquid // J. Appl. Mech. Tech. Phys. - 1966. - Vol. 7, no. 3. - P. 43.

42. Napolitano L. G. Plane Marangoni-Poiseulle flow of two immiscible // Acta Astronautica. 1980. - Vol. 7, no. 4. - P. 461-478.

43. Пухначёв В. В. Нестационарные аналоги решения Бириха // Изв. АлтГУ. - 2011. № $69(1 / 2)$. - С. 62-69.

44. Гончарова О. Н., Резанова Е. В. Пример точного решения стационарной задачи о двухслойных течениях при наличии испарения на границе раздела // Прикладная механика и техническая физика. - 2014. - № 2. - С. 68-79.

45. Aristov S. N., Prosviryakov E. Y. On laminar flows of planar free convection // Rus. J. Nonlin. Dyn. - 2013. - Vol. 9, no. 4. - P. 651-657. - DOI: 10.20537/nd1304004.

46. Schwarz K. G. Plane-parallel advective flow in a horizontal incompressible fluid layer with rigid boundaries // Fluid Dynamics. - 2014. - Vol. 49, iss. 4. - P. 438-442. DOI: $10.1134 / \mathrm{S} 0015462814040036$.

47. Bratsun D. A., Vyatkin V. A., Mukhamatullin A. R. On exact nonstationary solutions of equations of vibrational convection // Computational Continuum Mechanics. - 2017. - Vol. 10, no. 4. - P. 433-444. - DOI: 10.7242/1999-6691/2017.10.4.35.

48. Шварц К. Г. Плоскопараллельное адвективное течение в горизонтальном слое несжимаемой жидкости с внутренним линейным источником тепла // Прикладная математика и механика. - 2018. - Т. 82, № 1. - С. 25-30.

49. Aristov S. N., Shvarts K. G. Convective heat transfer in a locally heated plane incompressible fluid layer // Fluid Dynamics. - 2013. - Vol. 48, iss. 3. - P. 330-335. - DOI: 10.1134/S001546281303006X.

50. Горшков А. В., Просвиряков Е. Ю. Аналитические решения стационарной сложной конвекции, описывающие поле касательных напряжений разного знака // Труды института математики и механики УрО РАН. - 2017. - Т. 23, № 2. - С. 32-41.

51. Rafiq Sh., Nawaz M., Mustahsan M. Casson Fluid Flow due to Non-Coaxial Rotation of a Porous Disk and the Fluid at Infinity Through a Porous Medium // Journal of Applied Mechanics and Technical Physics. - 2018. - Vol. 59, iss. 4. - P. 601-607. - DOI: 10.1134/S0021894418040053.

52. Андреев В. К. Решения Бириха уравнений конвекции и некоторые его обобщения: препринт. - Красноярск, 2010. - № 1-10.

53. Birikh R. V. Thermocapillary convection in a horizontal layer of liquid // J. Appl. Mech. Tech. Phys. - 1966. - Vol. 7, no. 3. - P. 43-44.

54. Pukhnachev V. V. Group-theoretical methods in the convection problems // Application of Mathematics in Technical and Natural Sciences / ed. by M. D. Todorov and C. I. Christov. American Institute of Physics, CP 1404, Melwille, NY, 2011. - P. 31-42.

55. Sidorov A. F. Two classes of solutions of the fluid and gas mechanics equations and their connection to traveling wave theory // Journal of Applied Mechanics and Technical Physics. 1989. - Vol. 30, iss. 2. - P. 197-203. - DOI: 10.1007/BF00852164.

56. Aristov S. N., Shvarts K. G. Advective flow in a rotating liquid film // J. Appl. Mech. Tech. Phys. - 2016. - Vol. 57, no. 1. - P. 188-194. - DOI: 10.1134/S0021894416010211. 
57. Aristov S. N., Prosviryakov E. Yu. A new class of exact solutions for three-dimensional thermal diffusion equations // Theor. Found. Chem. Eng. - 2016. - Vol. 50, no. 3. - P. 286-293. DOI: $10.1134 /$ S0040579516030027.

58. Aristov S. N., Frik P. G. Nonlinear effects of the Ekman layer on the dynamics of largescale eddies in shallow water // J. Appl. Mech. Tech. Phys. - 1991. - Vol. 32, no. 2. - P. 189-194. DOI: $10.1007 / \mathrm{BF} 00858033$.

59. Аристов С. Н., Шварц К. Г. Вихревые течения адвективной природы во вращающемся слое жидкости. - Пермь : Пермский гос. ун-т, 2006. - 155 с.

60. Аристов С. Н., Шварц К. Г. Вихревые течения в тонких слоях жидкости. - Киров : Вятский гос. ун-т., 2011. - 207 с.

61. Ингель Л. Х., Аристов С. Н. Класс точных решений нелинейных задач о термических циркуляциях, связанных с объемным тепловыделением в атмосфере // Труды института экспериментальной метеорологи. - 1996. - № 27 (162). - С. 142-157.

62. Aristov S. N., Prosviryakov E. Yu. On laminar flows of planar free convection // Russian Journal of Nonlinear Dynamics. - 2013. - Vol. 9, no. 4. - P. 651-657. - DOI: 10.20537/nd1304004.

63. Mathematical Models of Convection / V. K. Andreev, Ya. A. Gaponenko, O. N. Goncharova, V. V. Pukhnachev. - Berlin, Boston : De Gryuter Publ., 2012. - 417 p.

64. Бекежанова В. Б., Гончарова О. Н. Задачи испарительной конвекции (обзор) // ПММ. 2018. - Т. 82, вып. 2. - С. 219-260.

65. Галкин В. А., Дубовик А. О. О моделировании тепловыделения в слоистом течении вязкой магнитной несжимаемой жидкости во вращающейся трубе // Вестник кибернетики. 2017. - № 4 (28). - С. 56-61.

66. Механика жидкости и газа. Избранное / под общей ред. А. Н. Крайко; ред.-сост. А. Н. Крайко, А. Б. Ватажин, Г. А. Любимов - М. : Физматлит, 2013. - 752 с.

67. Homogeneous condensation in turbulent submerged isobaric jets / A. B. Vatazhin, A. Yu. Klimenko, A. B. Lebedev, A. A. Sorokin // Fluid Dynamics. - 1988. - Vol. 23, iss. 2. P. 194-203. - DOI: 10.1007/BF01051887.

68. Vasil'kov A. P. Calculation of a turbulent two-phase isobaric stream // Fluid Dynamics. 1976. - Vol. 11, iss. 5. - P. 699-704. - DOI: 10.1007/BF01012960.

69. Shmiglevskiy $\mathrm{Yu}$. D. On isobaric plane flows of a viscous incompressible fluid // USSR Computational Mathematics and Mathematical Physics. - 1985. - Vol. 25, iss. 6. - P. 191-193. DOI: $10.1016 / 0041-5553(85) 90030-8$.

70. Шмыглевский Ю. Д. Аналитические исследования динамики газа и жидкости. М. : УРСС, 1999. - 232 c.

71. Koterov V. N., Shmiglevskiy Yu. D., Scheprov A. V. A survey of analytical studies of steady viscous incompressible flows (2000-2004) // Computational Mathematics and Mathematical Physics. - 2005. - Vol. 45, no. 5. - P. 867-888.

72. Aristov S. N., Prosviryakov E. Y. Inhomogeneous Couette flow // Russian Journal of Nonlinear Dynamics. - 2014. - Vol. 10, no. 2. - P. 177-182. - DOI: 10.20537/nd1402004.

73. Aristov S. N., Prosviryakov E. Y. Stokes waves in vortical fluid // Rus. J. Nonlin. Dyn. 2014. - Vol. 10, no. 3. - P. 309-318. - DOI: 10.20537/nd1403005.

74. Aristov S. N., Prosviryakov E. Y. Large-scale flows of viscous incompressible vortical fluid // Russian Aeronautics (IzVUZ). - 2015. - Vol. 58, no. 4. - P. 413-418. DOI: $10.3103 /$ S1068799815040091.

75. Aristov S. N., Prosviryakov E. Y. Unsteady layered vortical fluid flows // Fluid Dynamics. 2016. - Vol. 51, no. 2. - P. 148-154. - DOI: 10.1134/S0015462816020034. 
76. Aristov S. N., Knyazev D. V., Polyanin A. D. Exact solutions of the Navier-Stokes equations with the linear dependence of velocity components on two space variables // Theor. Found. Chem. Eng. - 2009. - Vol. 43, no. 5. - P. 642-662. - DOI: 10.1134/S0040579509050066.

77. Gershuni G. Z., Zhukhovitskii E. M. Instability of a system of horizontal layers of immiscible fluids heated from above // Fluid Dynamics. - 1980. - Vol. 15, iss. 6. - P. 816-822. DOI: $10.1007 / \mathrm{BF} 01096629$.

78. Фихтенгольц Г. М. Курс дифференциального и интегрального исчисления. Т. 1. М. : Физматлит, 2001. -616 с.

79. Кострикин А. И. Введение в алгебру. - Москва : Физматлит, 2004. - Ч. 1 : Основы алгебры. -272 с. 